\title{
Lesions in Posterior Parietal Area 5 in Monkeys Result in Rapid Behavioral and Cortical Plasticity
}

\author{
Jeffrey Padberg, ${ }^{1,2}$ Gregg Recanzone, ${ }^{2,3}$ James Engle, ${ }^{2,4}$ Dylan Cooke, ${ }^{2}$ Adam Goldring, ${ }^{2,4}$ and Leah Krubitzer ${ }^{2,4}$ \\ ${ }^{1}$ Department of Biology, University of Central Arkansas, Conway, Arkansas 72035, ${ }^{2}$ Center for Neuroscience, University of California Davis, Davis, \\ California 95618, and ${ }^{3}$ Departments of Neurobiology, Physiology and Behavior, and ${ }^{4}$ Psychology, University of California Davis, Davis, California 95616
}

We examined the effects of focal lesions of posterior parietal area 5 in macaque monkeys on bimanual behavior performed with and without visual guidance. The animals were trained on two reaching tasks and one tactile texture discrimination task. Task 1 simply involved reaching toward and grasping a reward from one of five well positions. Task 2 required the monkey to use both hands simultaneously to obtain a reward. The tactile texture discrimination task required the monkey to signal the roughness of a passively delivered texture using its jaw. After lesions to area 5, the monkeys showed a decrease in hand use for tasks 1 and 2 and an inability to perform task 2 in specific locations in visual space. These deficits recovered within several days. No deficits were observed in the tactile texture discrimination task or in an analgesic control monkey. Electrophysiological recordings made just before the lesion, immediately after the lesion, and 2 months after the lesion demonstrated that cortical areas just rostral to the lesioned area 5, and areas 1 and 2, were topographically reorganized and that receptive fields for neurons in these fields changed location on the body surface. These cortical map changes are correlative and may, in part, contribute to the rapid behavioral recovery observed. The mechanism for such rapid changes may be the unmasking of existing divergent and convergent thalamocortical connections that are part of the normal cortical circuitry.

\section{Introduction}

More than any other species, humans have evolved a remarkable ability to manipulate objects in the physical environment with their hands, which has resulted in an extraordinary transformation of our culture and our planet. This dexterity coevolved with an enormous expansion of the neocortex and an increase in the number of cortical areas associated with hand use and hand-eye coordination. Although manual abilities in nonhuman primates generally are not as sophisticated as those of humans, they are nonetheless impressive and rely upon similar neural mechanisms. Recent studies in both human and nonhuman primates demonstrate that most of the posterior parietal cortex is devoted to the coordination of the hands, reaching, grasping, and transforming sensory information into a coordinate system in which these actions can be initiated. (Ferraina and Bianchi, 1994; Lacquaniti et al., 1995; see Culham et al., 2003; 2006; Disbrow et al., 2007; Gardner, 2008; Hinkley et al., 2009 for review). Here we investigate the contribution of one such field in the posterior parietal cortex, area 5, to sophisticated bimanual behaviors.

Traditionally, Brodmann's area 5 assumed almost the entire extent of the rostral bank of the intraparietal sulcus (IPS), and portions of the lower caudal bank. However, current studies indicate that this traditional area 5 is one of several fields in this

Received April 8, 2010; revised July 15, 2010; accepted July 26, 2010.

This work was supported by National Institute of Neurological Disorders and Stroke Grant R01-NS35103 (to L.K.), National Eye Institute (NEI) Grant R01-EY013458 (to G.R.), and NEI Grant F32-EY014503-01A1 to (J.P.). We thank Adele Seelke for her help with the reconstruction of cortical maps, and Rebecca Grunewald for her help with histological processing of tissue.

Correspondence should be addressed to Leah Krubitzer, Center for Neuroscience, 1544 Newton Court, Davis, CA 95616. E-mail: lakrubitzer@ucdavis.edu.

DOI:10.1523/JNEUROSCI.1806-10.2010

Copyright $\odot 2010$ the authors $\quad 0270-6474 / 10 / 3012918-18 \$ 15.00 / 0$ sulcus including the parietal reach region (PRR) and the anterior intraparietal area (AIP), both of which are also involved in coordinated and visually guided reaching and grasping. Area 5 in a modern context refers to a more restricted area on the rostral bank of the IPS, which extends around the sulcal crown and onto the adjacent postcentral gyrus (Fig. 1, Table 1) (for review, see Iwamura et al., 2002; Gardner et al., 2007a; Gardner, 2008; Krubitzer and Disbrow, 2008), but does not include PRR. Much of area 5 is dominated by the representation of the forelimb (Taoka et al., 1998, 2000; Iwamura et al., 2002; Krubitzer and Disbrow, 2008), and neurons in area 5 respond during object grasping (e.g., Mountcastle et al., 1975). Recent studies demonstrate that neurons in area 5 increase their rate of firing as the fingers are preshaped just before grasping, and that object size and hand posture affect the firing rates of neurons (Gardner et al., 2007a,b). These studies support previous studies that indicate that area 5 is involved in programming the intention of movement (Kalaska, 1996; Snyder et al., 1997; Debowy et al., 2001), and also that visual input is an important component for motor planning in area 5. Despite the emerging view of area 5 as a critical component in a sensory motor network for complex manual behaviors, no previous study has examined the direct effects of focal lesions to area 5 on these manual abilities.

In the present study, our objectives were as follows: (1) to examine how lesions restricted to area 5 affect reaching, grasping, and bilateral coordination of the hands with and without visual input; (2) to determine the time course of functional recovery after lesions to area 5; and (3) to examine the postlesion functional organization in cortical areas adjacent to area 5 to determine whether reorganization of these fields could ultimately underlie behavioral recovery. 


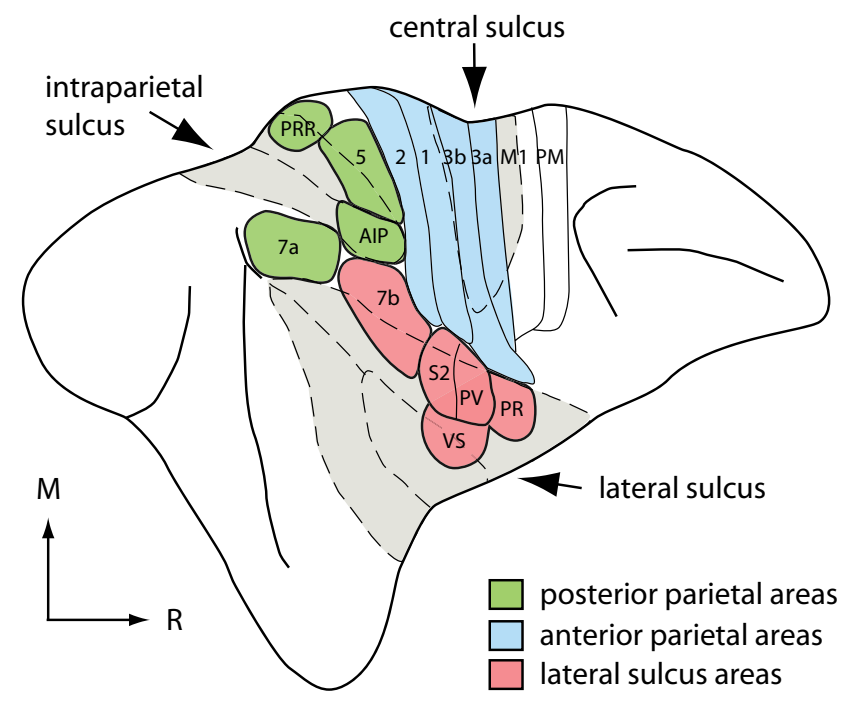

Figure 1. The location and extent of somatosensory areas in anterior parietal cortex (blue), posterior parietal cortex (green), and lateral sulcus (pink). Areas in anterior parietal cortex process inputs from cutaneous and deep receptors of the skin, muscle, and joints. Although area 5 is driven by stimulation of somatic receptors, neurons here also respond to visual stimulation. Neurons in areas 5 and AIP are active during reaching and grasping in awake animals. Solid lines mark architectonic boundaries, and dashed lines mark sulci that have been reflected to display areas on the banks of the sulci. Abbreviations are defined in Table 1.

\section{Materials and Methods}

Five adult male macaque monkeys (Macaca mulatta) were used in these studies. Two animals (cases A and B; 13.6 and $12.5 \mathrm{~kg}$, respectively) were used to examine the effects of lesions of cortical area 5 on visually and non-visually guided reaching and grasping, and the extent to which adjacent, spared cortex is reorganized. Two other monkeys (cases C and D; 8.4 and $8.3 \mathrm{~kg}$, respectively) were used to determine the detailed functional organization of areas 1 and 2 in an intact brain, and to compare receptive fields (RFs) for neurons at different time points. One monkey (case AC; $14.5 \mathrm{~kg}$ ) was used as a control to determine the effects of postoperative analgesics on all behavioral tasks. All of the procedures used in these studies were approved by the Institutional Animal Care and Use Committee and conformed to National Institutes of Health guidelines for the care and use of animals.

Animals used for lesions and behavioral testing were selected from a larger pool of monkeys at the California National Primate Research Center based on two criteria. First, the monkeys were required to have all functioning fingers on both hands. Second, the monkeys had to be tractable, and willing to be touched by the investigator. We also tested the monkeys for hand preference (Welles, 1976; Hörster and Ettlinger, 1985), although the hand preference was not a selection criterion. To test for handedness, the monkeys were given the option to use one or the other hand in a grasping task that required the animal to reach and grasp a reward located $\pm 60^{\circ}, 30^{\circ}$, and $0^{\circ}$ in horizontal space. A "handedness index" was calculated as the number of trials using the dominant hand divided by the number of trials tested, restricted to locations $-30^{\circ}, 0^{\circ}$, and $+30^{\circ}$. Pretesting in six macaque monkeys indicated one-third (33\%) displayed preferential handedness (i.e., used one hand $>80 \%$ of the time). One of the monkeys (case A) was left handed, and the other monkey was ambidextrous with only a slight preference for the right hand (case B). The analgesic control monkey was ambidextrous (case AC).

\section{Behavioral methods}

Two behavioral testing devices ("boxes") and one control device were used to assess reaching and grasping, bimanual coordination, and tactile discrimination abilities. All devices were assembled from quarter-inch acrylic and polycarbonate sheeting and PVC (polyvinylchloride) tubing. Microswitches, photocells, and light-emitting diodes (LEDs) were controlled by a computer via a digital input/output interface card (Acces I/O Products). All sessions were recorded on video for post hoc off-line analysis.

\section{Table 1. List of abbreviations}

\begin{tabular}{|c|c|}
\hline Cortical areas & Cortical regions \\
\hline 1 & Somatosensory area 1 \\
\hline 2 & Somatosensory area 2 \\
\hline $3 a$ & Somatosensory area 3a \\
\hline $3 b$ & Primary somatosensory cortex \\
\hline 5 & Cortical area 5 \\
\hline $7 a$ & Cortical area 7a \\
\hline $7 \mathrm{~b}$ & Cortical area $7 \mathrm{~b}$ \\
\hline AIP & Anterior intraparietal area \\
\hline M1 & Primary motor cortex \\
\hline M & Motor cortex \\
\hline PM & Premotor cortex \\
\hline PR & Rostroventral parietal area \\
\hline PV & Parietal ventral area \\
\hline S2 & Secondary somatosensory cortex \\
\hline VS & Ventral somatosensory area \\
\hline \multicolumn{2}{|r|}{ restra } \\
\hline CS & Central sulcus \\
\hline IPS & Intraparietal sulcus \\
\hline LS & Lateral sulcus \\
\hline PCS & Postcentral sulcus \\
\hline \multicolumn{2}{|c|}{ Anatomical directions } \\
\hline D & Dorsal \\
\hline L & Lateral \\
\hline M & Medial \\
\hline $\mathrm{R}$ & Rostral \\
\hline \multicolumn{2}{|c|}{ Body part representations } \\
\hline Bilat & Bilateral \\
\hline Bil wr & Bilateral wrist \\
\hline d & Distal \\
\hline $\operatorname{dig}$ & Digits \\
\hline dor & Dorsal \\
\hline D1-D5 & Digits 1 through 5 \\
\hline el & Elbow \\
\hline $\mathrm{fa}$ & Forearm \\
\hline $\mathrm{fl}$ & Forelimb \\
\hline hypoth & Hypothenar pad \\
\hline ipsi & Ipsilateral \\
\hline $\mathrm{m}$ & Middle \\
\hline pads & Glabrous pads \\
\hline prox & Proximal \\
\hline p1-p5 & Glabrous pads 1 and 2 \\
\hline rf & Receptive field \\
\hline sh & Shoulder \\
\hline th & Thenar pad \\
\hline ven & Ventral \\
\hline wr & Wrist \\
\hline
\end{tabular}

Each box was initially placed onto the home cage of the monkey for sessions of $\sim 30 \mathrm{~min}$ each to acclimatize the monkey to the box. During each of these sessions, the monkey received food rewards for interacting with the box (e.g., pressing the levers). On subsequent sessions, the method of successive approximations was used until the monkey was performing the necessary sequence of actions to successfully complete trials with each box. Once the monkey was performing each task, daily training sessions of $30-100$ trials were run until $90 \%$ of trials were successfully completed (except for one monkey on the nonreaching, nonvisual texture discrimination task). A trial was considered a success if the monkey retrieved the reward within $15 \mathrm{~s}$. Once trained, however, the monkeys completed the vast majority of trials within a few seconds.

Rewards consisted of primate chow-based flavored pellets (Bio-Serv) and fresh or dried fruit/vegetable pieces. Each monkey's feeding schedule was monitored and adjusted throughout the training period under the recommendations of the veterinary staff to keep the animal motivated to work diligently and to maintain body weight within $10 \%$ of the original weight. During the perilesion behavioral testing ( $10 \mathrm{~d}$ prelesion and $10 \mathrm{~d}$ 


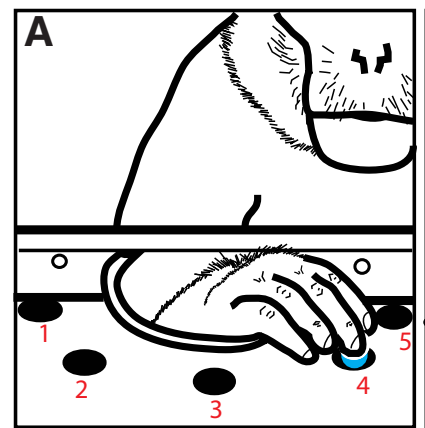

visual -location 4 -right hand first attempt -retrieved

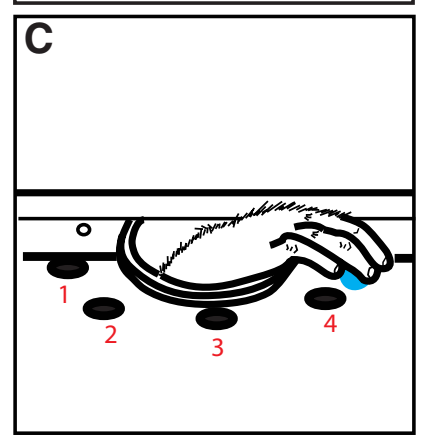

non-visual -location 4 right hand-first attempt retrieved

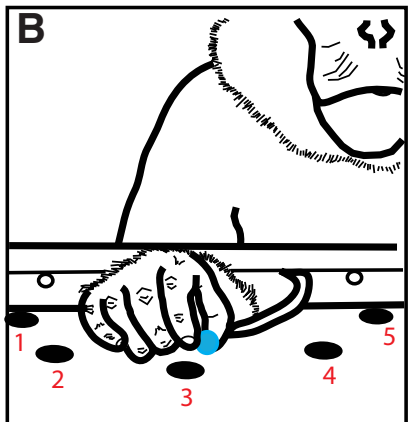

visual - location 3 -right hand first attempt- retrieved

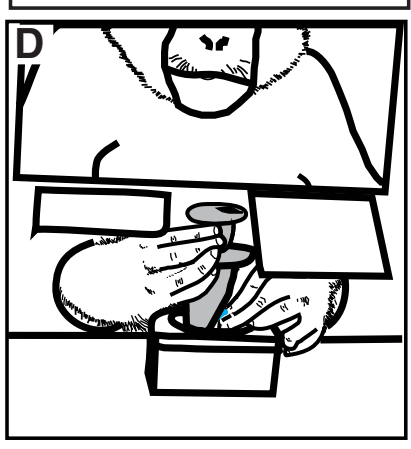

visual -location L- rt lift hand It retrieval hand-stereotypic first attempt -retrieved

Figure 2. $\quad \boldsymbol{A}-\boldsymbol{D}, \mathrm{A}$ monkey performing task $1(\boldsymbol{A}-\boldsymbol{C})$ and task 2 (D) under different conditions. For task 1 , the monkey was required to retrieve a reward from one of five well locations under visual $(\boldsymbol{A}, \boldsymbol{B})$ or nonvisual $(\boldsymbol{C})$ guidance. Some of the scoring conditions are listed in the panels below the figures. For task $2(\boldsymbol{D})$, in this trial the monkey performed the task under visual guidance, the reward location was left, the hand used to retrieve was left, and the grip was precision. For videotape scoring, see supplemental Tables 1 and 2 (available at www.jneurosci. org as supplemental material).

postlesion), the normal feedings were delayed until immediately after the testing sessions were completed.

Once a criterion of $90 \%$ success was achieved, performance was maintained with the animal performing $60-70$ trials on task 1 and $50-60$ trials on task 2 per session. During testing in the $10 \mathrm{~d}$ before and after the lesion, this was increased to two sessions on each task per day for a total of 130-140 trials for task 1, 100-120 for task 2, and 100 trials for the tactile texture discrimination task. The day-to-day order in which these three tasks were presented as well as the order of trials within each task on a given day were randomized.

\section{Task 1: reaching and grasping}

For task 1 (Fig. 2A-C), the monkeys faced a window where an opaque screen was raised or lowered on alternating trials by the experimenter to reveal or occlude the view of the food wells. A food reward was then placed on top of a photocell at one of five well locations by the experimenter. A "go" signal to start a trial was a blinking red LED. The monkeys were required to to depress a left and right start lever with the left and right hands, respectively, which resulted in the blinking LED being illuminated continuously. For visual trials (screen raised), once the start levers had been depressed for $\sim 500 \mathrm{~ms}$ and the screen was raised, a green LED was illuminated, signaling that the monkey could now reach into the box with one hand to retrieve the reward. For nonvisual trials, once the start levers were depressed the opaque screen was lowered and then the green go LED was illuminated. The monkey was not forced to use one hand over the other to retrieve the reward, but was allowed to use whatever hand he preferred. Reward locations were presented pseudorandomly with no more than

two consecutive trials at the same well location such that all conditions were presented the same number of times.

\section{Task 2: bimanual coordination}

For task 2, the monkeys were required to face a clear window into the apparatus (Fig. 2D) and place each hand onto one of two start levers in response to a blinking red LED. For all trials, the reward was loaded atop a photocell inside a well on a small vertically oriented cylinder with two small disks in view of the monkey (Fig. $2 D$ ). The top disk on the cylinder aided the monkey in grasping, and the bottom disk, just above the reward hole, prevented the cylinder from dropping too far at the completion of the task. Depending on the trial type, an opaque screen was then lowered or raised (nonvisual, lowered; visual, raised), and the trial was started. Once the start levers had been depressed for $\sim 500 \mathrm{~ms}$, the green LED was illuminated and the monkey reached into the apparatus with both hands to raise the cylinder with one hand, exposing the food well, and retrieve the reward from the well with the other hand, necessitating the simultaneous use of both hands. The cylinder was presented with the reward well in one of three positions (left, center, and right, from the monkey's perspective) that changed randomly across the trials in each session. As in the first task, the monkey was not required to use one hand over the other to perform any aspect of this task, but could freely use either hand to perform different aspects of this task.

\section{Texture discrimination task: rough/smooth discrimination}

To rule out general effects of surgery, including reduced motivation, cognitive abilities and general motor control, the monkeys were required to perform a texture discrimination task that should not be affected by a lesion to area 5 , as it did not require reaching or visual input. This nonreaching, nonvisual texture discrimination task was performed with the monkey in a primate chair, allowing the experimenters safe access to the animal's arm. A movable PVC rod with a rough texture on one end and a smooth texture on the other end was mounted horizontally within reach of the monkey. A small opaque shield was placed on the front of the chair to prevent the monkeys from seeing the textured rod. To start a trial, the monkey's hand was lifted off the rod by the experimenter, and the rod was randomly switched from one texture to the other texture (several switches were always made between trials). The hand was then replaced on the rod, and the rod was spun one half turn, allowing the monkey to experience the texture passively. A red LED then cued the monkey to indicate whether a rough or smooth texture was detected. The monkey then turned its head and depressed a lever on the left (smooth) or right (rough) using its jaw. Thus, no reaching movements were necessary to perform this task. Monkeys were rewarded for correct responses with food pellets delivered though a tube near the lever, and the experimenter recorded responses. A minimum performance of $70 \%$ correct was the required criterion for each monkey. Two additional texture rods with alternate texture configurations were randomly and occasionally tested to probe whether texture was the salient cue for the monkey (rather than location), and the data from these probes always supported that texture was the cue. It should be noted that the monkeys took several months longer to reach criteria for this task than tasks 1 and 2, indicating that the task was relatively difficult compared with tasks 1 and 2 .

\section{Behavioral data analysis}

Performance for each of the two target tasks was evaluated in two ways. The first was latency to perform the task, and the second was a frame-by-frame video analysis to quantify accuracy, success, and other aspects of the manual behavior. Performance on the nonreaching, nonvisual texture discrimination task was evaluated using percentage correct across responses.

Latency. Response time for tasks 1 and 2 were recorded as the time the green LED was illuminated until the monkey removed its hands from the levers. The overall latency was recorded as the time between green LED illumination and reward removal from the well (Tables 2, 3). For task 2, latency to the initial lifting of the cylinder, and latency to the cylinder returning to the start position were also recorded.

Scoring of videotapes. In addition to latency, videos taken between $10 \mathrm{~d}$ before and $10 \mathrm{~d}$ after the lesion were analyzed using a scoring system to quantify details of hand and digit configuration, and success on each trial. To establish inter-rater reliability, two individuals were instructed 
Table 2. Hand use and latency data for three tasks in monkey A and monkey B

\begin{tabular}{|c|c|c|c|c|c|c|c|c|c|c|c|c|c|}
\hline \multirow[b]{3}{*}{ Case } & & & \multirow[b]{3}{*}{ Condition } & \multicolumn{4}{|l|}{ Task $1^{a}$} & \multicolumn{6}{|l|}{ Task $2^{b}$} \\
\hline & \multicolumn{2}{|c|}{$\begin{array}{l}\text { Somato } \\
\text { task LH } \\
\text { (\% correct) }\end{array}$} & & \multicolumn{2}{|l|}{$\begin{array}{l}\text { Completed } \\
\text { trials } \mathrm{L} / \mathrm{R} \\
(\% \mathrm{~L} / \mathrm{R})\end{array}$} & \multicolumn{2}{|l|}{$\begin{array}{l}\text { Latencies } \\
\text { L/R } \\
\text { (SD L/R) }\end{array}$} & \multicolumn{2}{|c|}{$\begin{array}{l}\text { Trials retrieved } \\
\text { with left } \\
\text { hand (\%) }\end{array}$} & \multicolumn{2}{|c|}{$\begin{array}{l}\text { Mean } \\
\text { latency (s) }\end{array}$} & \multicolumn{2}{|c|}{$\begin{array}{l}\text { Trials } \\
\text { completed } \\
\text { (No.) }\end{array}$} \\
\hline & Pre & Post & & Pre & Post & Pre & Post & Pre & Post & Pre & Post & Pre & Post \\
\hline \multirow[t]{6}{*}{ Monkey A } & 70 & 70 & V & $49 / 15.3$ & $21 / 17$ & $0.91 / 1.1$ & $1.3^{*} / 1.6$ & L: 100 & L: 100 & $\mathrm{~L}: 2.9$ & L: 5.2 & 90 & 14 \\
\hline & & & & $(76 / 24)$ & $(55 / 45)$ & $(0.21 / 0.19)$ & $(0.39 / 0.90)$ & C: 100 & C: 100 & $C: 3.7$ & $C: 7.0$ & & \\
\hline & & & & & & & & $\mathrm{R}: 0$ & $\mathrm{R}: 0$ & $\mathrm{R}: 2.7$ & $\mathrm{R}: 6.0$ & & \\
\hline & & & NV & $62.3 / 5$ & $32 / 15$ & $1.1 / 1.1$ & $1.4^{*} / 1.6^{*}$ & L: 99 & $\mathrm{~L}: 0$ & L: 4.4 & $\mathrm{~L}:-$ & & \\
\hline & & & & $(93 / 7)$ & $(68 / 32)$ & $(0.30 / 0.33)$ & $(0.32 / 0.44)$ & C: 98 & $C: 0$ & $C: 5.7$ & $C: 3.6$ & & \\
\hline & & & & & & & & $\mathrm{R}: 2$ & $\mathrm{R}: 0$ & R: 5.0 & $\mathrm{R}: 3.1$ & & \\
\hline \multirow[t]{6}{*}{ Monkey B } & 89 & 85 & V & $26.4 / 37.5$ & $4 / 8$ & 1.7/1.6 & 2.0/1.8 & L: 100 & L: 100 & $\mathrm{~L}: 3.1$ & L: 3.3 & 91.3 & 26 \\
\hline & & & & $(41 / 59)$ & $(33 / 67)$ & $(0.35 / 0.4)$ & $(0.53 / 0.31)$ & C: 100 & C: 0 & C: 3.9 & $C: 2.5$ & & \\
\hline & & & & & & & & $\mathrm{R}: 2$ & $\mathrm{R}: 0$ & $\mathrm{R}: 3.7$ & $\mathrm{R}: 3.7$ & & \\
\hline & & & NV & $25.8 / 39.6$ & $4 / 3$ & $1.5 / 1.5$ & $1.7 / 1.9$ & L: 94 & L: 100 & $\mathrm{~L}: 3.4$ & L: 5.9 & & \\
\hline & & & & $(39 / 61)$ & $(57 / 43)$ & $(0.59 / 0.43)$ & $(0.65 / 1.4)$ & C: 100 & C: 100 & $C: 3.9$ & $C: 4.5$ & & \\
\hline & & & & & & & & $\mathrm{R}: 0$ & $\mathrm{R}: 0$ & R: 4.3 & $\mathrm{R}: 6.9$ & & \\
\hline
\end{tabular}

Values are given as means. V, Visual; NV, nonvisual; Pre, prelesion; Post, postlesion; L, left; C, center; R, right; Somato, somatosensory; - , data from left location in first postlesion session in task 2 for monkey A was not collected because of a sensor malfunction. * Significant difference.

${ }^{a}$ For task 1, the first three postlesion sessions were merged (see Latencies subsection).

${ }^{b}$ Postlatency means for task 2 are for first session postlesion only, with deficit gone by fourth/fifth day postlesion.

Table 3. Hand use and latency data for three tasks in monkey AC

\begin{tabular}{|c|c|c|c|c|c|c|c|c|c|c|c|c|c|c|c|c|c|c|}
\hline \multirow{2}{*}{\multicolumn{3}{|c|}{$\begin{array}{l}\text { Somato task LH } \\
\text { (\% correct) }\end{array}$}} & \multirow[b]{3}{*}{ Condition } & \multicolumn{6}{|l|}{ Task 1} & \multicolumn{9}{|l|}{ Task 2} \\
\hline & & & & \multicolumn{3}{|c|}{$\begin{array}{l}\text { Completed trials } \mathrm{L} / \mathrm{R} \\
(\% \mathrm{~L} / \mathrm{R})\end{array}$} & \multicolumn{3}{|c|}{$\begin{array}{l}\text { Latency L/R } \\
\text { (SD L/R) }\end{array}$} & \multicolumn{3}{|c|}{$\begin{array}{l}\text { Trials retrieved with } \\
\text { left hand (\%) }\end{array}$} & \multicolumn{3}{|c|}{ Mean latency (s) } & \multicolumn{3}{|c|}{$\begin{array}{l}\text { Trials } \\
\text { completed }\end{array}$} \\
\hline Pre & Drug & Post & & Pre & Drug & Post & Pre & Drug** & Post ${ }^{* *}$ & Pre & Drug & Post & Pre & Drug & Post & Pre & Drug & Post \\
\hline \multirow[t]{6}{*}{91} & 93 & 97 & V & $14 / 19$ & $16 / 15$ & $13.5 / 17.5$ & $0.8 / 0.76$ & $1.0 / 0.9$ & $1.0 / 0.9$ & L: 100 & L: 100 & L: 100 & L: 5.1 & $\mathrm{~L}: 5.7$ & L: 4.9 & 31.5 & 31 & 30 \\
\hline & & & & $(42 / 58)$ & $(52 / 48)$ & $(44 / 56)$ & $(0.1 / 0.05)$ & $(0.23 / 0.1)$ & $(0.12 / 0.04)$ & $C: 77$ & C: 91 & C: 67.6 & $C: 5.3$ & $C: 7.3^{*}$ & $C: 6.1$ & & & \\
\hline & & & & & & & & & & $\mathrm{R}: 0$ & $R: 4.5$ & $\mathrm{R}: 0$ & $R: 5.1$ & $\mathrm{R}: 8.6^{*}$ & $\mathrm{R}: 6.7^{*}$ & & & \\
\hline & & & NV & $22 / 16$ & $21.3 / 16.3$ & $16.5 / 23$ & $0.89 / 0.76$ & $1.2 / 1.1$ & $1.3 / 1.24$ & L: 88 & L: 89 & L: 94.5 & L: 5.2 & $\mathrm{~L}: 6.5$ & L: 4.8 & & & \\
\hline & & & & $(58 / 42)$ & $(57 / 43)$ & $(42 / 58)$ & $(0.1 / 0.11)$ & $(0.28 / 0.11)$ & $(0.21 / 0.14)$ & C: 70 & $C: 85$ & C: 58 & $C: 8.6$ & C: 10.9 & C: 9.6 & & & \\
\hline & & & & & & & & & & $\mathrm{R}: 0$ & $R: 0$ & $\mathrm{R}: 0$ & R: 4.9 & $\mathrm{R}: 6.9^{*}$ & R: 5.6 & & & \\
\hline
\end{tabular}

Values are given as means. V, Visual; NV, nonvisual; Pre, predrug; Post, postdrug; L, left; C, center; R, right; Somato, somatosensory. *Significant difference; **During and after analgesia, means were significantly different to the means before analgesia when visual and nonvisual conditions were grouped together.

in the rating system described below and then coded the videotapes of both experimental tasks. The exemplar videos were the same for each rater in training and had been chosen at random from the entire selection of behavioral videotapes. Both individuals scored the same 60 trials for each video, and these scores were compared against the same 60 trials that had been coded by the other rater. The criteria for establishing reliability required the two individuals to agree on $\geq 95 \%$ of the trials. The animal condition (prelesion or postlesion) was blind to the individuals coding the data.

A number of different parameters for tasks 1 and 2 were recorded by the scorers. For task 1, the condition (visual vs nonvisual), reward location, hand used (left or right), accuracy (monkey reaching into correct/ incorrect well), number of attempts, retrieval success, and hand posture when reaching for and retrieving the reward were all scored and quantified for prelesion and postlesion conditions for both monkeys (supplemental Table 1, available at www.jneurosci.org as supplemental material) (Fig. 2). For task 2, we examined condition (visual vs nonvisual), reward location (left/center/right), first hand used to lift the tower (left or right), number of hand switches when lifting the tower, reach and grasp posture, hand used to retrieve the reward, digits used to retrieve the reward, first well location attempted (left/center/right), the number of attempts, and success in retrieval (supplemental Table 2, available at www.jneurosci. org as supplemental material). For tasks 1 and 2, all parameters were individually analyzed by mixed ANOVA with Bonferroni adjustments. However, statistical analysis was not possible in the first $5 \mathrm{~d}$ after the lesion because neither monkey would perform the task on postlesion days 1 and 2, and both would only perform a limited number of trials for each task on postlesion days 3-5.

\section{Electrophysiological recording procedures}

Standard electrophysiological recording procedures were used to record from cortex before the lesion to determine the site where the lesion should be made. In one case, immediately after the lesion electrophysiological recordings were also made. Finally, electrophysiological recordings were made in both cases $62-70 \mathrm{~d}$ after the lesion. The anesthetic and surgical protocols were the same for both the short-term and long-term recording sessions. For recovery procedures, the cortex was bathed in sterile saline, and for terminal mapping experiments the cortex was covered with silicone fluid to prevent desiccation. Recordings were made with low-impedance, coated tungsten microelectrodes designed to record from single neurons or neuron clusters (1-5 M $\Omega$ at $1 \mathrm{kHz}$; A-M Systems). The neural response was amplified (A-M Systems), filtered, monitored through a loudspeaker, and viewed on an oscilloscope. For electrophysiological recordings on the dorsal surface of the cortex in areas 1 and 2, the electrode was positioned manually with a micromanipulator (Kopf Instruments), and for recordings in the sulcus a stepping hydraulic microdrive (Kopf Instruments) was used to advance the electrode in $500 \mu \mathrm{m}$ increments. For short-term recording experiments, the recording sites were sparsely distributed over a large cortical area, because the goal of these experiments was to rapidly identify area 5 and surrounding cortex. All recordings were made in the middle cortical layers, $700-1000 \mu \mathrm{m}$ from the pial surface. Recording depth was later verified in histologically processed tissue. For terminal mapping experiments in both lesioned monkeys, areas 1 and 2 were mapped in detail, and hundreds of closely spaced recording sites were obtained for each case. Cutaneous stimuli used to elicit responses from neurons at each site included fine probes, brushes, and hair displacement. Deep receptors of 


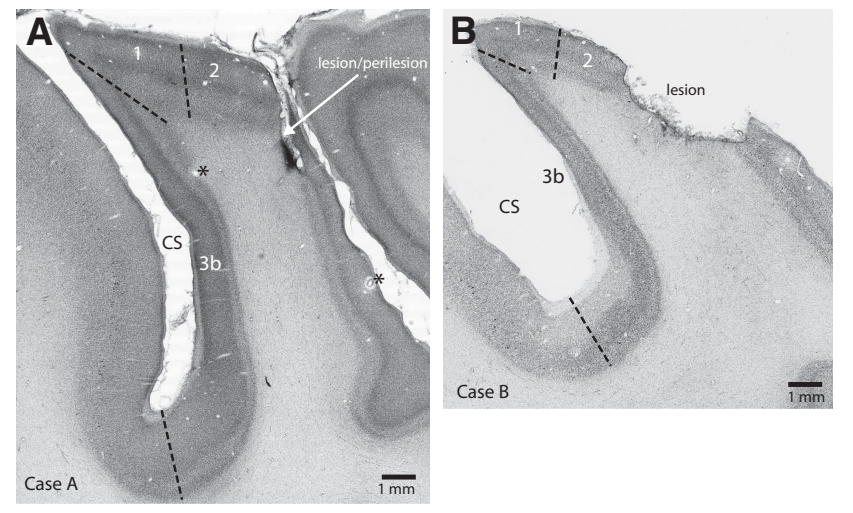

Figure 3. $A, B$, Horizontal sections that have been stained for Nissl in case $A(A)$ and case $B$ $(\boldsymbol{B})$. In case $A$, the walls of the lesion were pushed toward the center. In case $B$, the area of the lesion was clearly visible since the walls of the lesioned area did not push in to fill the lesioned space after the recovery period. The white matter below area 5 was not significantly affected by the lesion and was only superficially involved. The boundaries of areas 2 and 1 could still be identified. Thin dashed lines mark architectonic boundaries. Abbreviations are defined in Table 1.

the skin and muscle were stimulated by light to hard taps, muscle squeezes, and joint manipulation. Full field flashes of light and bars of light were used for visual stimulation. These methods are similar to those used previously in our own laboratory and those of others (Pons et al., 1985; Padberg et al., 2009). Receptive fields for neurons at each site were noted in a written record and marked on drawings of the body. For all cases, small electrolytic lesions ( $5 \mu \mathrm{A}$ for $10 \mathrm{~s}$ ) and probes were placed at strategic locations for later identification in histologically processed tissue.

\section{Identification of cortical fields}

For lesion experiments, electrophysiological recording techniques were used to rapidly identify cortical areas on the postcentral gyrus and the rostral bank of the IPS. Using a simple set of criteria, it was relatively easy to rapidly identify areas 1, 2, and 5 in the absence of architectonic data. First, the size of receptive fields generally varies between these fields. Neurons in area 1 have very small receptive fields that are highly localized to a small portion of the body, such as a small portion of the distal tip or hairy hand (a few digits). Receptive fields for neurons in area 2 are larger and can encompass an entire glabrous digit. Finally, receptive fields for neurons in area 5 are often quite large and may encompass an entire hand or both hands. Another criterion used to identify the field is the modality of stimulation to which neurons respond. Neurons in area 1 respond almost exclusively to cutaneous stimulation, and neurons in area 2 respond to cutaneous stimulation or stimulation of deep receptors of the skin and joints. Neurons in area 5 respond to muscle and joint manipulation, hard squeezes to the hand, and in many instances visual stimulation. A final criterion was the responsiveness of neurons under our recording conditions. Neurons in areas 1 and 2 respond well to the types of stimulation described above, whereas responsiveness of neurons in area 5 in an anesthetized preparation varies from good (although less vigorous) to unresponsive. Properties of neurons in areas 1 and 2 have been well described previously in other laboratories using a similar preparation (Nelson et al., 1980; Pons et al., 1985) Properties of neurons in area 5 under our or similar recording conditions have been described for macaque monkeys (Taoka et al., 1998, 2000; Krubitzer and Disbrow, 2008; Padberg et al., 2009), cebus monkeys (Padberg et al., 2007), and titi monkeys (Padberg et al., 2005). Using these criteria, we were able to identify area 5 and make a lesion that was limited to its boundaries. We subsequently confirmed the lesion location in histological preparations of tissue from both of these animals (Fig. 3).

\section{Surgical procedures}

Anesthesia was induced with ketamine hydrochloride (10 mg/kg, i.m.). Once anesthetized, the animals were intubated and cannulated, and maintained at surgical levels of anesthesia on the inhalant anesthetic isoflurane (1-2\%). The animals were continuously infused with lactated Ringer's solution $(6 \mathrm{ml} / \mathrm{kg} / \mathrm{h}$, i.v. $)$, and respiration rate, heart rate, expired $\mathrm{CO}_{2}$ levels, and temperature were monitored throughout the experiment. Atropine $(0.4 \mathrm{mg} /$ kg, i.m.), doxapram hydrochloride (Dopram; $0.4 \mathrm{mg} / \mathrm{kg}$, i.m.), and dexamethasone $(0.4 \mathrm{mg} / \mathrm{kg}$, i.m. $)$ were also administered at the beginning of the experiment. For recovery (lesion) experiments in which the animals recovered, cefazolin $(20 \mathrm{mg} / \mathrm{kg}$, i.m. ) was administered intraoperatively and postoperatively for $5 \mathrm{~d}$, and oxymorphone $(0.15 \mathrm{mg} / \mathrm{kg}$, i.m. $)$ was administered postoperatively 3 times/d for $2-3 \mathrm{~d}$.

Once the animal was anesthetized, the skin was cut, the temporal muscle was slightly retracted, and a craniotomy was made over cortical area 5 and adjacent cortex. The dura was cut and retracted, and the cortex was exposed. A digital image was made of the cortex so that recording sites could be plotted relative to vascular patterns. For recovery experiments, all procedures were performed under sterile conditions.

For lesion experiments, 23-52 recording sites were made in areas 5, 1, and 2. In one monkey, case B, neurons in area 5 were unresponsive. Thus, the area to be lesioned was determined from the sulcal pattern. The relationship of the sulcus to the hand representation in area 1 (where neurons were responsive) also helped us estimate the mediolateral extent of area 5. Boundaries were then confirmed post hoc using an architectonic analysis (see below, Data analysis for lesion reconstruction, functional map organization, and receptive field comparisons). The location of the intended lesion was marked on our digital image for guidance during cortical aspiration. In both monkeys, the right hemisphere was lesioned. Lesions were made by gently aspirating cortex in the lateral portion of the rostral bank of the IPS, while leaving the cortex on the caudal bank of the IPS intact. Care was taken not to destroy underlying white matter (Fig. 3). Upon completion of the lesion, in one monkey (case A) electrophysiological recordings were made in cortex adjacent to the lesion, in areas 2 and 1, and receptive fields were defined. Once the recordings were complete, the cortex was covered with a soft contact lens, the dura flaps were pulled over the contact lens, and then the craniotomy was closed with an acrylic skull cap. After the animal recovered from anesthesia ( $30 \mathrm{~min}-2 \mathrm{~h}$ after surgery), it was returned to its home cage. These experiments were kept to a maximum time of $4 \mathrm{~h}$ from initial opening of the skin until suturing of the skin.

One monkey (case AC) served as a control for the effects of analgesics after surgery. The behavior of this animal on all tasks was recorded for $2 \mathrm{~d}$ before administration of analgesics, during the administration of oxymorphone $(0.15 \mathrm{mg} / \mathrm{kg}$, i.m.; 3 times per day for $3 \mathrm{~d})$, and for $3 \mathrm{~d}$ after the administration of oxymorphone. The same doses and administration schedule of oxymorphone used for lesion experiments was implemented. This monkey was part of a separate set of experiments, and the number of days of formal testing and the number of trials were necessarily limited by the monkey's special feeding regime. Thus, a minimum of 120 trials of task 1,90 trials of task 2, and 100 trials of the nonreaching, nonvisual texture discrimination task were run each day, and the total number of days for all testing was $8 \mathrm{~d}$.

\section{Histological preparation of tissue}

After the terminal mapping experiments were complete, the animals were killed with sodium pentobarbital $(60 \mathrm{mg} / \mathrm{kg}$, i.v.) and then transcardially perfused with $0.9 \%$ saline followed by $4 \%$ paraformaldehyde in phosphate buffer, and then $4 \%$ paraformaldehyde in $10 \%$ sucrose in phosphate buffer, $\mathrm{pH}$ 7.4. The brain was removed from the skull, weighed, and photographed from multiple angles. After $\sim 36 \mathrm{~h}$ immersion in the final perfusate, the lesioned brains were cut horizontally into $60 \mu \mathrm{m}$ sections. Images of the tissue block were taken before every section of the brain was cut. After the brain was sectioned, alternate series were stained for Nissl substance (Fig. 3) or processed for cytochrome oxidase (Wong-Riley, 1979). One of the brains of the two monkeys used for determining the functional organization of areas 1 and 2 was cut horizontally into $80 \mu \mathrm{m}$ sections and processed as described above. For the second normal brain, the cortex was removed from the brainstem, the sulci were peeled apart, and the cortex was flattened and cut into $50 \mu \mathrm{m}$ sections tangential to the pial surface. A one in three series of sections was stained for myelin (Gallyas, 1979).

\section{Data analysis for lesion reconstruction, functional map organization, and receptive field comparisons}

For the two brains that contained area 5 lesions, histological data were analyzed as follows. Architectonic boundaries, tissue artifacts, small electrolytic lesions, probes, and electrode tracts were drawn onto a digital image of the block face that corresponded to the histologically processed 


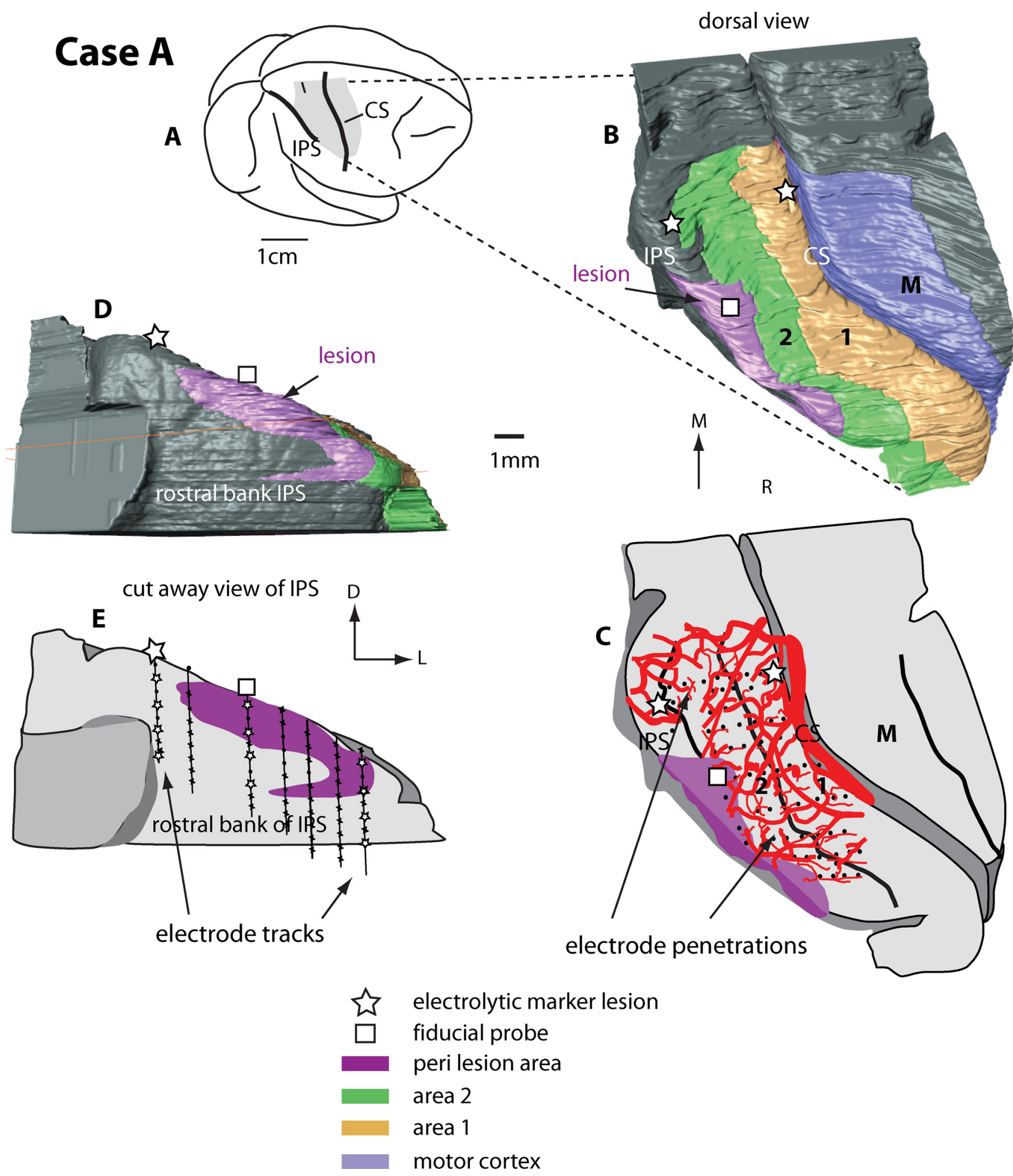

Figure 4. $A-E$, An example of $3 D$ data generation and analysis from case $A$. The region of the cortex studied in detail is depicted as the gray shaded region in $A$. A $3 D$ blowup of this region is illustrated in $B$. This illustration includes architectonically defined cortical areas (green, gold and purple), the perilesion area (dark pink), the location of fiducial probes (square), electrolytic marker lesions (stars), and sulci. An illustration of this $3 \mathrm{D}$ reconstruction is shown below in $\boldsymbol{C}$, and contains the features in illustration $\boldsymbol{B}$, plus the vascular pattern and electrophysiological recording sites. The Amira software used to generate this $3 \mathrm{D}$ reconstruction allows us the rotate the reconstruction in any plane and to digitally "cut away" sulcal walls. The 3D reconstruction shown in D is a view of the rostral bank of the intraparietal sulcus with the posterior bank cut away. This shows the location of the perilesion region into the depth of the sulcus and as well as the location of the fiducial probe and an electrolytic lesion made during the final mapping session. An illustration of this $3 \mathrm{D}$ reconstruction is shown in $\boldsymbol{E}$, which also contains electrode tracks (and their angle) into the IPS as well as recording sites along those tracks. Abbreviations are defined in Table 1.

section. In addition, the area where cortex was removed, and the perilesion area was also drawn. In one case, cortex that composed the perimeter of the lesion remained separated through the $62 \mathrm{~d}$ after the lesion (case B) (Fig. 3B). In the other case, the cortex that composed the perimeter of the lesion came together so that no region where cortex was absent could be identified in gross brain examination (case A) (Fig. 3A). These reconstructions were performed for the entire series of sections that contained the lesion and maps of areas 1 and 2. The digital block-face images that 
contained the data described above were loaded into a computer, and using the Amira software program (Visage Imaging), were combined into a three-dimensional (3D) reconstruction of the cortex that could be rotated and viewed in any orientation on the computer screen (Fig. 4B,D). This system allows us to "digitally cut away" sulcal walls to expose the opposite sulcal wall and determine the angle of our electrode within a particular sulcus (Fig. 4D,E). Using the Amira software program, we could calculate the volume of each lesion and/or the perilesioned area.

To make functional maps of the cortex, drawings of the digital image taken before mapping were made and included recording sites, vasculature, and location of probes. The brain photograph with its map of recording sites was aligned with the $3 \mathrm{D}$ reconstruction of the cortical sections by matching the locations of fluorescent probes and marker lesions (Fig. 4C). Electrode entry points for sulcal recording were then matched with the recording tracks on the 3D reconstruction by aligning probes, and this allowed us to reconstruct recording sites within the sulcus (Fig. 4E). In this way, a comprehensive reconstruction containing recording sites, blood vessels, architectonic boundaries, lesion, and probes could be generated.

To compare prelesion and long-term postlesion recording sites, digital images of the brain taken before the lesion, immediately after the lesion, and 62 or $70 \mathrm{~d}$ after the lesion were precisely aligned using large and medium blood vessels, sulcal patterns, and the lesion itself to align both maps (Fig. 5). The vascular pattern for large blood vessels was remarkably consistent over the 2 month period after the lesion. Prelesion and postlesion recording sites could thus be matched with a great deal of accuracy. Once the maps were precisely aligned, receptive fields for neurons in the same location, or within a $250-400 \mu \mathrm{m}$ radius of each other could be directly compared.

This method was similar to comparisons made in studies of plasticity by Merzenich et al. (1983; see their Fig. 1). Receptive fields that were obtained for these paired recording sites were compared by calculating the amount of skin or body part shared by the receptive field for neurons at each site. We termed this measure the "overlap index," which is expressed as a percentage. For instance, recording sites that contained neurons that had the identical receptive field had an overlap index of $100 \%$; those that had no shared portion of the body or skin had an overlap index of $0 \%$, and those that had some overlap had an overlap index with a value between 100 and $0 \%$. Receptive field drawings made during recording sessions were scanned and digitally traced, and areas were measured using Adobe Illustrator software. A similar process was used to measure areas within functional maps.

The procedures of functional map reconstruction have been well described previously by our own and other laboratories (Pons et al.,1985; Padberg et al., 2005, 2007). In this study, the amount of cortex devoted to the representation of the glabrous hand and palm was measured in prelesion, postlesion, and normal maps using Adobe Illustrator software, and these area measures were expressed in square millimeters.

\section{Results}

\section{Anatomical identification of lesion}

Using Amira software, we were able to combine results from histologically processed tissue and electrophysiological recordings onto the block-face images of the brain to generate a threedimensional reconstruction of the cortex that contained the vascular pattern, electrophysiological recording sites, electrode
0-60 minutes post lesion
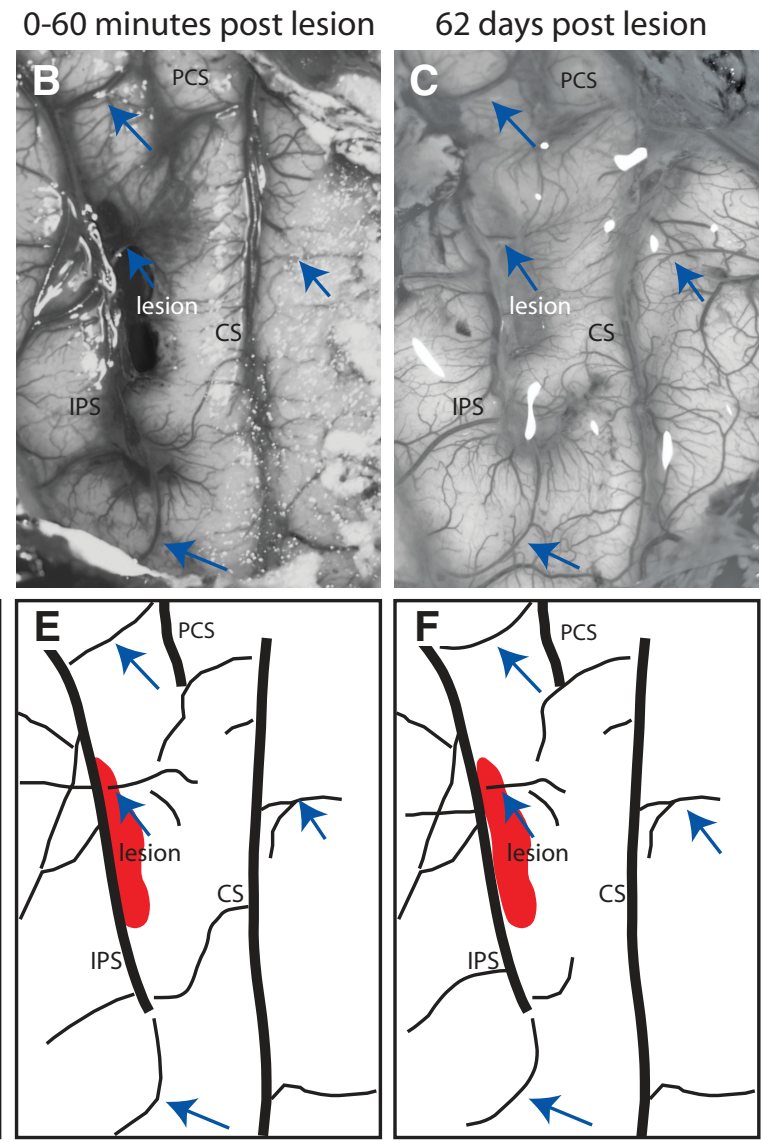

Case B

Figure 5. Alignment of digital images of the exposed neocortex before and after the lesion to area 5. $A-F$, Digital image and an tration of the cortex taken before $(\boldsymbol{A}, \boldsymbol{D})$, immediately after $(\boldsymbol{B}, \boldsymbol{E})$, and $62 \mathrm{~d}$ after $(\boldsymbol{C}, \boldsymbol{F})$ a lesion to area 5 in case B. Major sulc itself also served to hep align iod could be compared across time points. The lesion is denoted in red in $\boldsymbol{E}$ and $\boldsymbol{F}$. Abbreviations are defined in Table 1.

tracks, architectonic boundaries, and the perilesioned area in both monkeys (Figs. 3-5). As depicted in Figure 3, in both lesion cases, neighboring cortical areas 2 and 1 could be readily identified and had a normal laminar organization. In one case for which the lesioned area was readily identified (case B) (Fig. 3B), it is clear that our lesions did not encroach significantly on underlying white matter, although there was a small superficial involvement of white matter in this case. In the other monkey (case A), the lesion walls were pushed in by surrounding cortex so that the lesion itself was only visible as a small slit in the cortex (Fig. 3A). The underlying white matter in this case did not appear to be included in the lesion. It is not possible to calculate the true extent of either lesion since the pressure exerted on the lesion walls from the spared cortex pushed the walls of the lesion toward the center, collapsing entirely in one case. However, using Amira software we did calculate the region that was either absent or somewhat disorganized. In case A, this affected volume was 27.2 $\mathrm{mm}^{3}$, and in case B was $27 \mathrm{~mm}^{3}$.

\section{General observations after lesions to area 5 in the right hemisphere}

One monkey (case B) was tested on task $124 \mathrm{~h}$ before and after the lesion, and the other monkey (case A) was tested on task 1 $24 \mathrm{~h}$ before the lesion and $72 \mathrm{~h}$ after the lesion. Although testing was delayed after the lesion, the behavior of these monkeys outside of the formal testing sessions was observed. Within the first 
Control Task: Texture discrimination

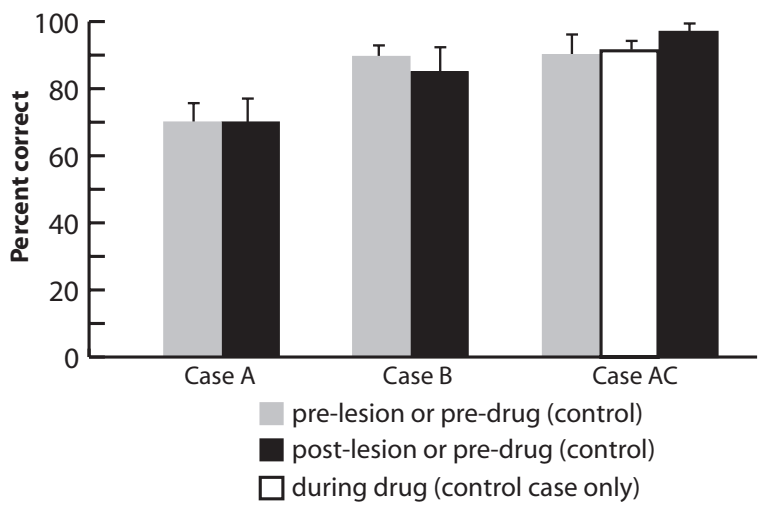

Figure 6. Results for the two area 5 lesioned monkeys (cases A and B), and the analgesic control monkey (case AC) on a rough vs. smooth somatosensory discrimination task using the left (contralesional) hand. There was no statistically significant difference in accuracy on this task between prelesion versus postlesion sessions $(p>0.05)$ difference in performance on this task in either lesioned monkey or in the analgesic control monkey.

$24 \mathrm{~h}$ postsurgery, both monkeys were able to retrieve small reward pellets from forage boards and from the experimenters' hands, often using a precision grip with the thumb and forefinger of the contralesional hand. Locomotion was seemingly unaffected by the lesion, with one monkey using the hand to grasp the bars of the cage when climbing and descending. Both monkeys recovered rapidly from the surgery, and lethargy and lack of appetite were no longer evident by 36-48 $\mathrm{h}$ postlesion. The monkey that demonstrated a clear lefthand preference (case A) used the contralesional and dominant hand (left) more slowly and deliberately for retrieving rewards in the first $48 \mathrm{~h}$ postlesion relative to prelesion. This latter observation was confirmed in our formal postlesion testing, which began on postlesion day 3. After the lesion, the monkeys were continually coaxed to perform each of the tasks during two sessions a day for a period of $\sim 60-90 \mathrm{~min}$. The control monkey, which underwent testing before drug administration, during the administration of oxymorphone, and after oxymorphone delivery, showed no noticeable signs of clumsiness or an inability to perform any of the tasks.

\section{Texture discrimination task}

The nonreaching, nonvisual texture discrimination task was designed to ensure that the animal would perform a tactually based task that should not be influenced by a lesion of area 5 . Although the monkeys completed few task 1 and 2 trials in the days after the lesion, the number of nonreaching, nonvisual texture discrimination task trials was not impacted. Importantly, initiation of a head movement to signal the decision was not affected by the lesions to area 5, indicating that the monkey was equally motivated to perform the task in prelesion and postlesion conditions. There was no statistically significant difference in the animals' ability to discriminate between a smooth versus a rough surface before and after the lesion ( $p>0.05$ ) (Fig. 6). Before the lesion, monkey A could distinguish rough and smooth textures with a mean accuracy of $70 \%$, and monkey B with a mean accuracy of $89 \%$. After the lesion, the animals made these same discriminations with equal accuracy, even in the first testing day after the lesion (day 3). The retention of this ability, and to do so over a relatively large number of trials ( 100 per session) after the lesions, suggests that the deficits seen in the other two tasks were not attributable to a lack of motivation, attention, motor or cognitive abilities, or some other generalized effect related to the surgical procedures themselves.
Task 1: Hand Use
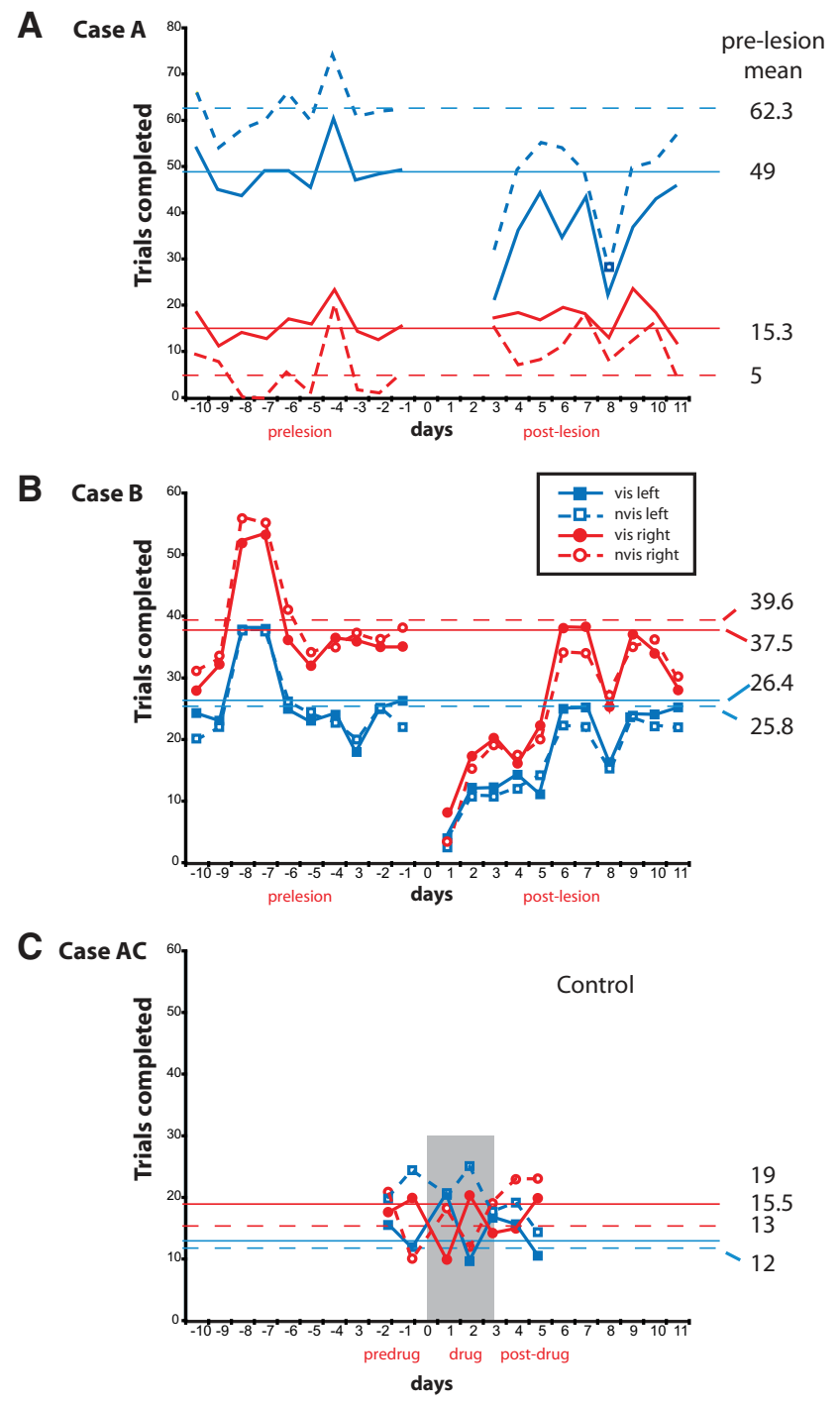

Figure 7. $A-C$, The mean daily hand use for task 1 collapsed across conditions and wells before and after lesions [cases $A(\boldsymbol{A})$ and $B(\boldsymbol{B})$ ] and before, during, and after analgesic administration [case $\mathrm{AC}(\boldsymbol{C})$ ]. The right hemisphere in both monkeys in $\boldsymbol{A}$ and $\boldsymbol{B}$ had the area 5 lesion. After the lesion, the use of the left hand dropped dramatically for this task for the lefted monkey (case A), and in the ambidextrous monkey (case B) the use of both hands dropped dramatically. Hand use in the analgesic control monkey (case $\mathrm{AC}$ ) was unchanged during the administration of drugs for $3 \mathrm{~d}$ (gray shaded region). Solid blue lines indicate left hand/visual condition; dashed blue lines indicate left hand/nonvisual condition; solid red lines indicate right hand/visual condition; and dashed red lines indicate right hand/nonvisual condition. Prelesion and preanalgesic means are shown at the far right.

The monkey that served as the analgesic control (case AC) (Fig. 6) performed 100 trials during all phases of the test with a similar mean accuracy ( 93\%) (Tables 2, 3) before, during, and after the administration of oxymorphone. There were no statistically significant differences during the three conditions, indicating that analgesics did not affect the performance on this tactile discrimination task ( $t$ test with Bonferroni correction, $p>0.05$ ).

Task 1: visually and non-visually guided reaching and grasping The prelesion/preanalgesic behavior on our monkeys was highly consistent (Fig. 7). All monkeys performed the task rapidly, and performed all or most of the trials. The prelesion mean number of trials performed with the contralesional (left) hand in both visual and nonvisual conditions for case $\mathrm{A}$, case $\mathrm{B}$, and the analgesic 


\section{Task 2: Performance: I}
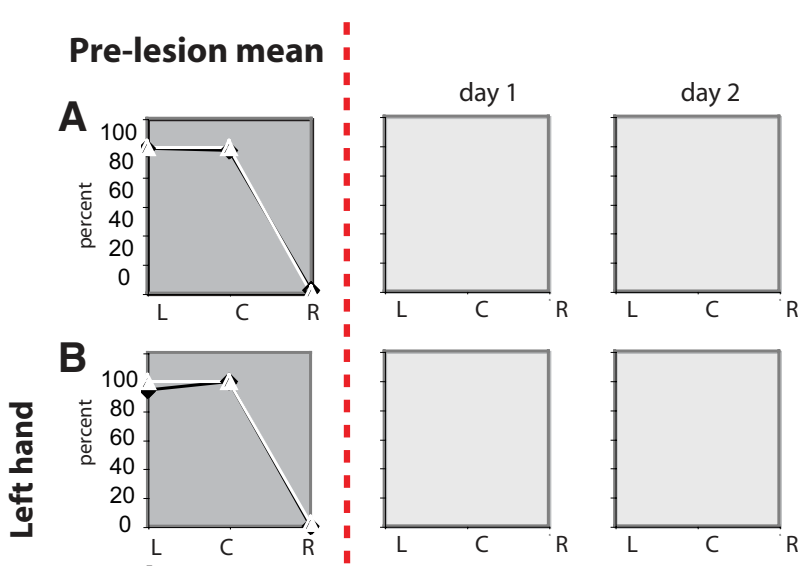

\section{Post lesion}
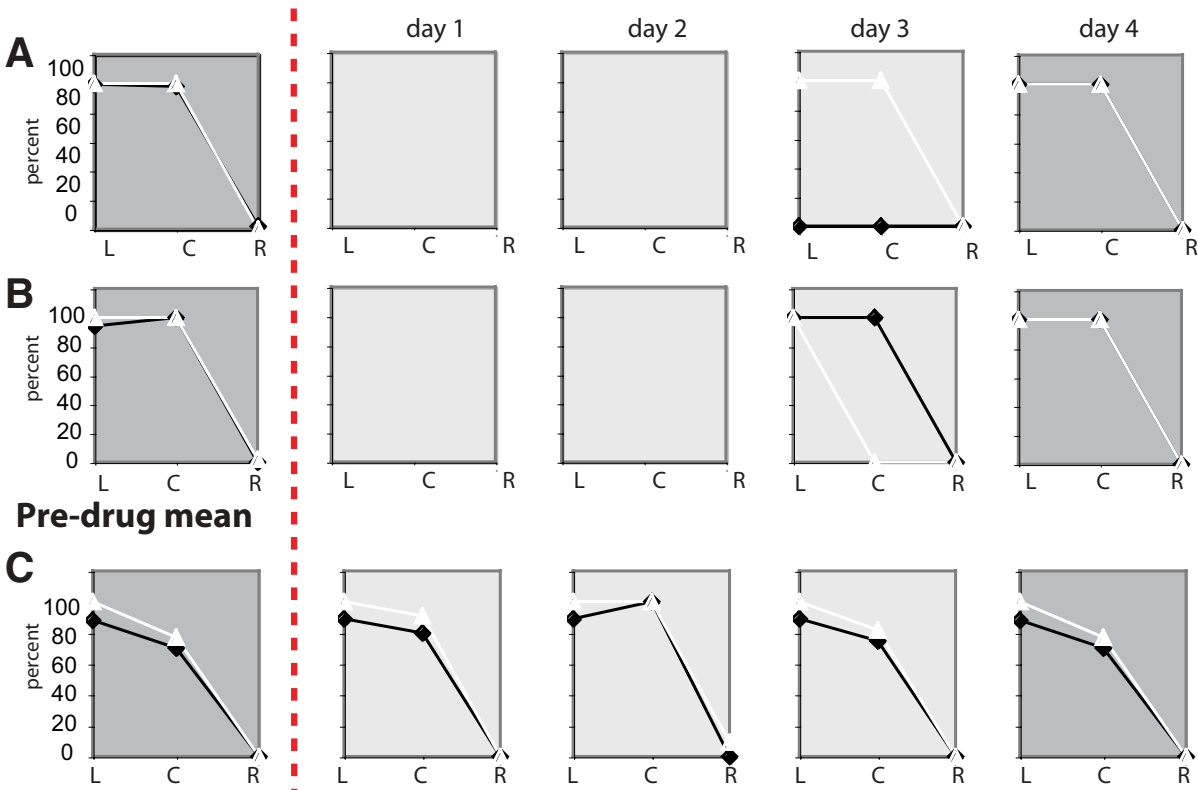

\section{Pre-lesion mean}
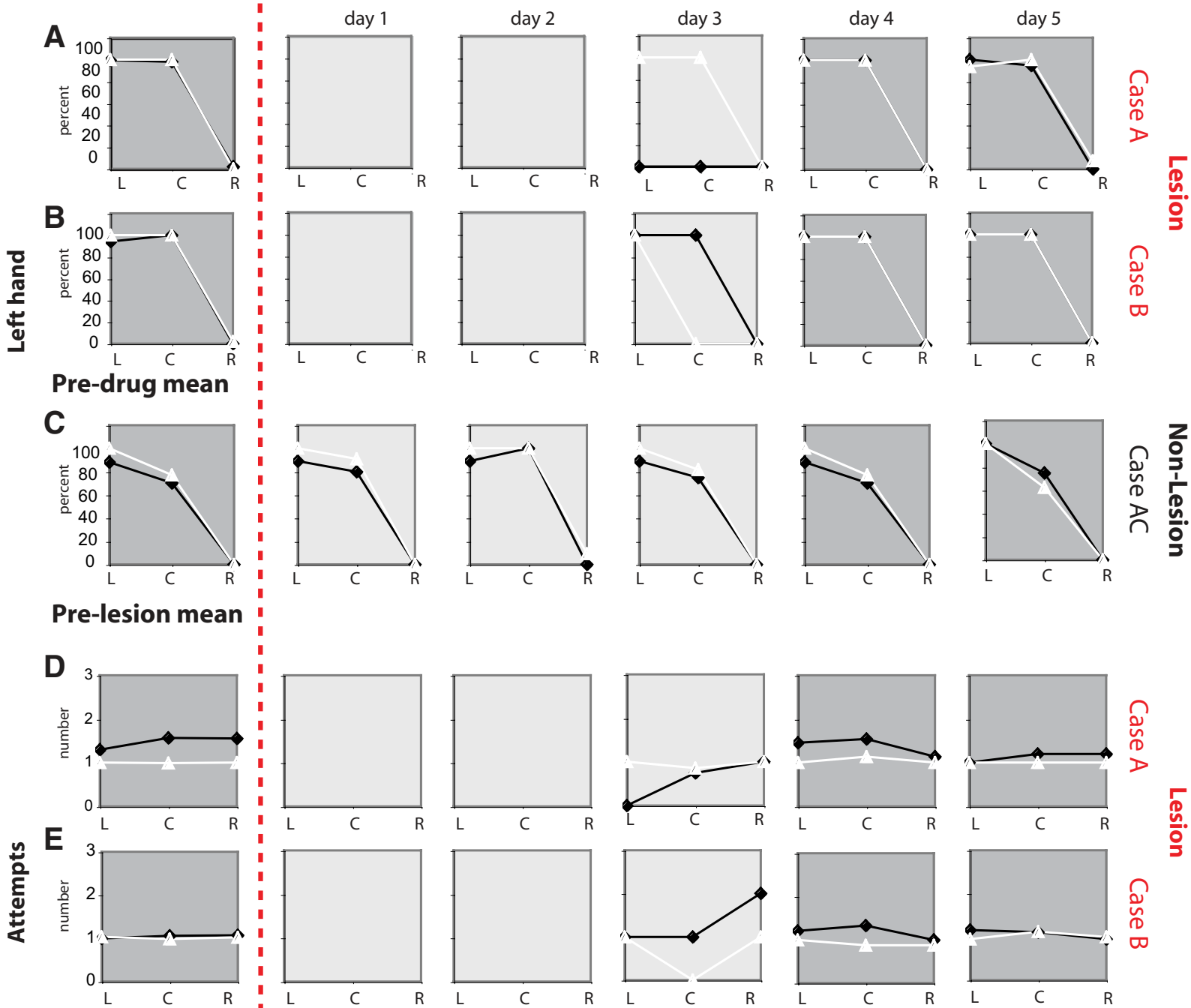

Pre-drug mean
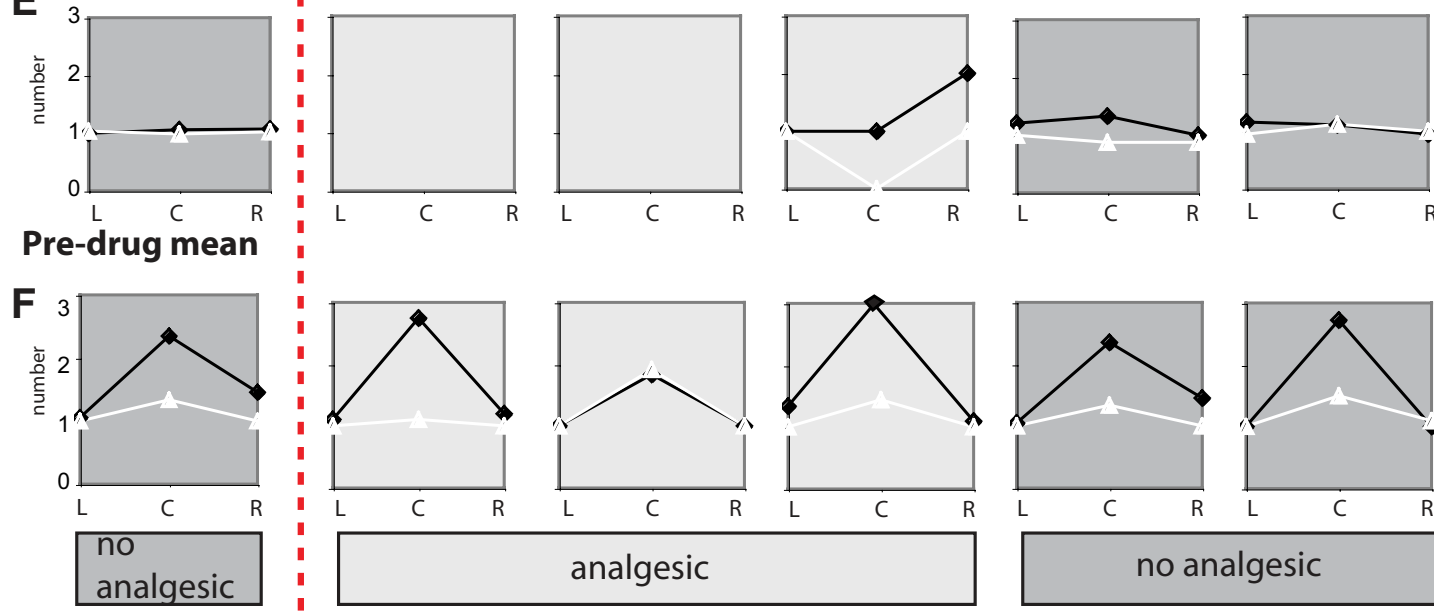

$\frac{\sqrt{6}}{\frac{h_{0}}{3}}$

lesion/drug delivery

analgesic
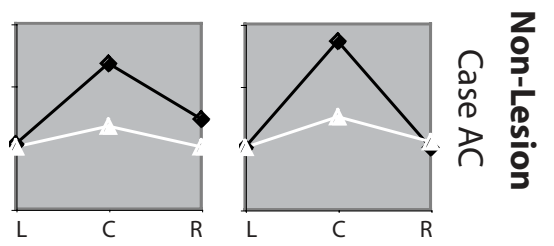

\section{no analgesic}

Visual condition $\triangle \quad$ Non-visual condition

Figure 8. $A-F$, Performance on task 2 for the two lesioned monkeys $(\boldsymbol{A}, \boldsymbol{B}, \boldsymbol{D}, \boldsymbol{E})$ and the analgesic control $(\boldsymbol{C}, \boldsymbol{F})$ on both the use of the contralesion left hand to retrieve the reward $(\boldsymbol{A}-\boldsymbol{C})$ and the number of attempts $(\boldsymbol{D}-\boldsymbol{F})$. The lesioned monkeys were unable to perform the task the first $2 \mathrm{~d}$ after the lesion while the analgesic control monkey performed the task normally $(\boldsymbol{C}, \boldsymbol{F})$. On the third postlesion day of testing, the left-handed monkey [case $A(A)$ ] did not use the contralesional left hand to retrieve the reward from the left or central location in the nonvisual condition, while case $B$ did not use the contralesion hand to retrieve the treat from the central location under visual guidance $(\boldsymbol{B})$. Both lesioned monkeys could not complete the task for retrieval $(\boldsymbol{D}, \boldsymbol{E})$ or increased the number of attempts for retrieval $(\boldsymbol{E})$ on the third day after the lesion. For both lesioned monkeys performance returned to normal by the fourth or fifth postlesion day.

control (case AC) is shown in Figure 7, and values are shown in Tables 2 and 3. Because the number of trials that each monkey would perform for this task during the first postlesion testing days was very low, statistical tests of significance were not performed on data from these days.
Hand use

One of the parameters that changed dramatically after the lesion was the number of trials performed with each hand. After the lesion, the number of reaching and grasping trials performed by each monkey dropped dramatically in the first few days com- 


\section{Task 2: Performance: II}
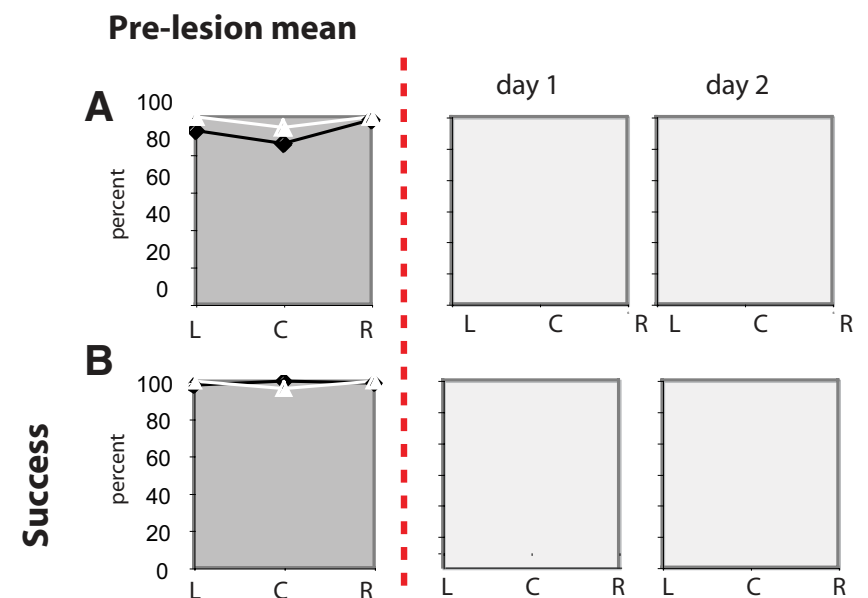

Post lesion

\section{Pre-drug mean}
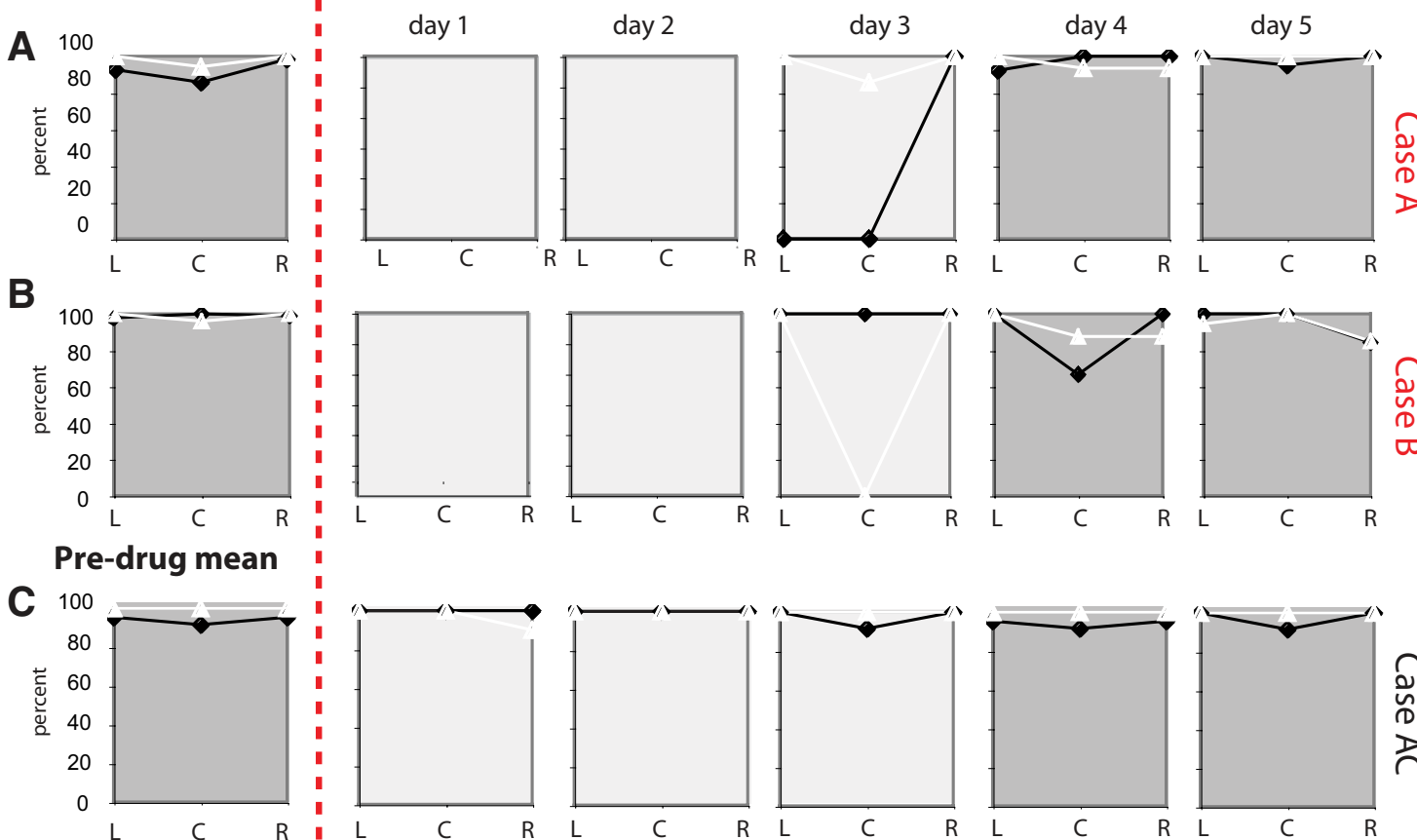

옹.
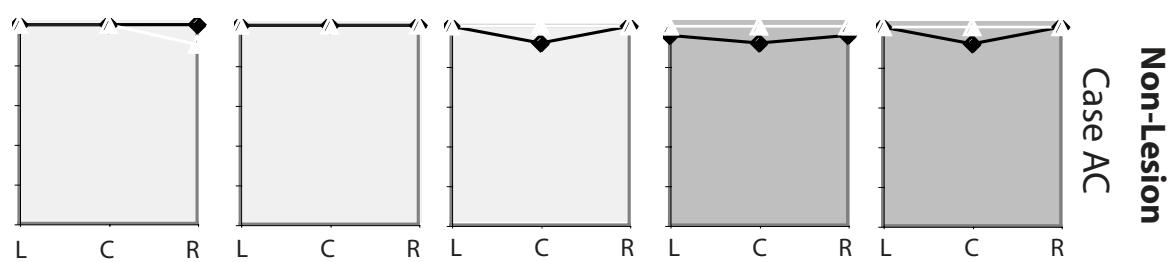

\section{Pre-lesion mean}
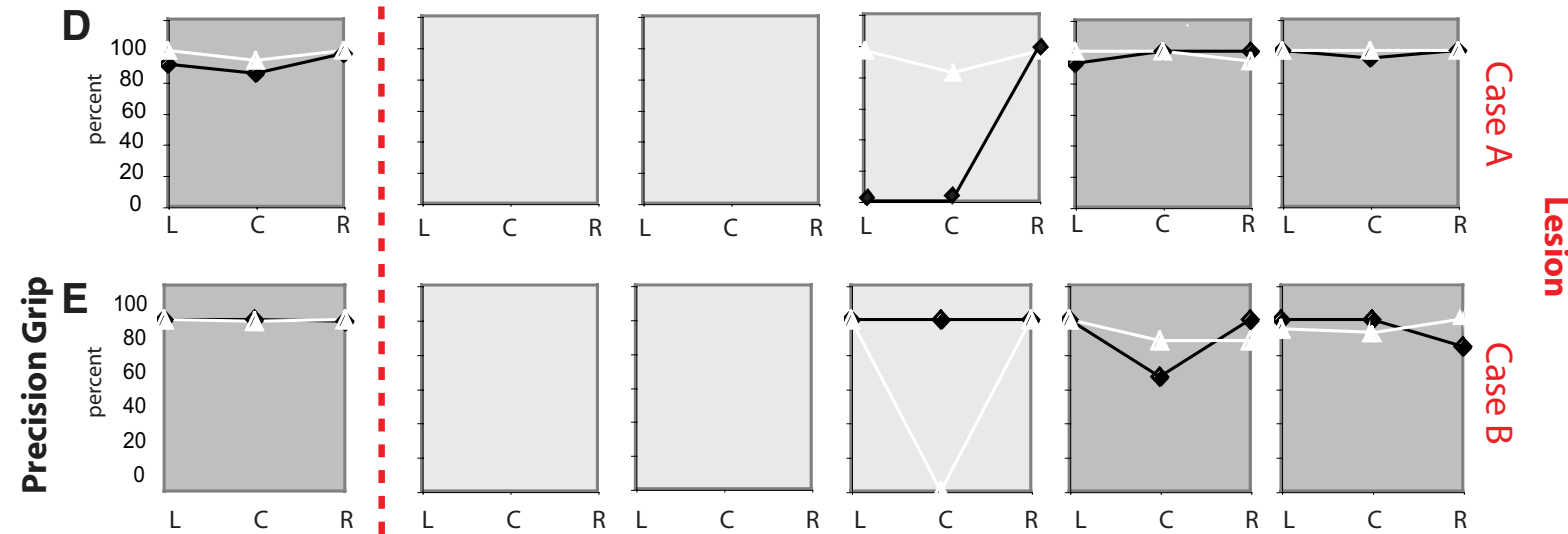

\section{ํㅗㅇ.}

Pre-drug mean
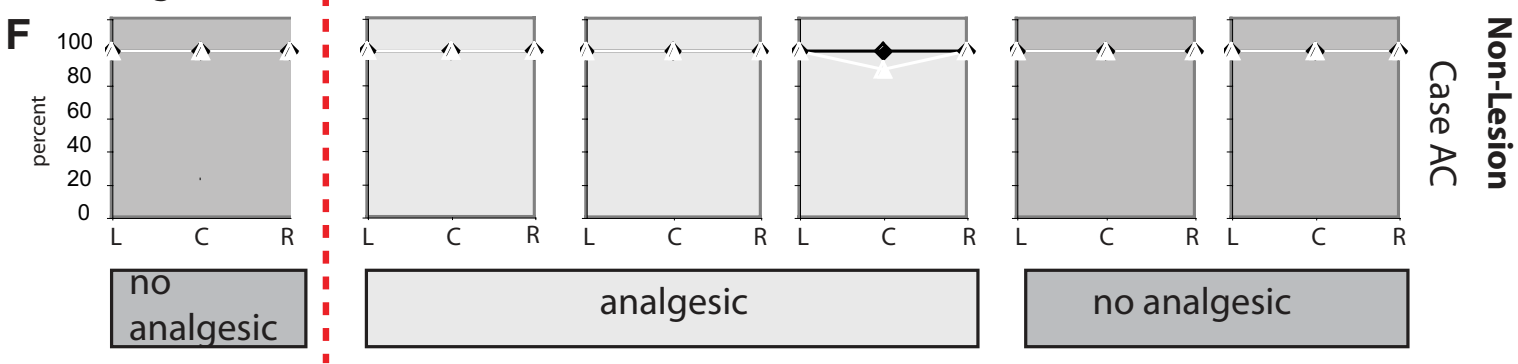

lesion/drug delivery

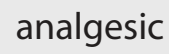

no analgesic

\section{Visual condition $\triangle$ Non-visual condition}

Figure 9. $A-F$, Performance on task 2 for the two lesioned monkeys $(\boldsymbol{A}, \boldsymbol{B}, \boldsymbol{D}, \boldsymbol{E})$ and the analgesic control $(\boldsymbol{C}, \boldsymbol{F})$ for success rate $(\boldsymbol{A}-\boldsymbol{C})$ and the use of the precision grip when retrieving a reward $(\boldsymbol{D}-\boldsymbol{F})$. Lesioned monkeys were unable to perform the task in the first $2 \mathrm{~d}$ after the lesion while the analgesic control monkey performed the task normally throughout the administration of the analgesic $(\boldsymbol{C}, \boldsymbol{F})$. The success rate for the left-handed monkey [case $A(\boldsymbol{A})$ ] dropped for the left and center well location while the second monkey (case B) had a poor success rate for the central well location compared with prelesion behavior. Further, the use of the precision grip changed for both lesioned monkeys $(\boldsymbol{D}, \boldsymbol{E})$. 
pared with the prelesion mean. The monkey with the left-hand preference (case A) completed an average of 49 trials using the left hand under visual guidance before the lesion; $72 \mathrm{~h}$ postlesion he completed only 21 trials with this contralesional hand under the same conditions. Likewise, nonvisual left-hand trials dropped from a mean of 62.3-32 trials (Fig. 7A). In contrast, use of the right (nondominant) hand on visually guided trials actually increased during this time period from 15.3 prelesion to 17 postlesion, and the use of the right hand for the nonvisual condition increased from a mean of $5-15$ trials. The ability of the monkey to perform the normal number of trials with the ipsilesional, nondominant hand $72 \mathrm{~h}$ postlesion indicated that general surgical effects were unlikely the cause of the drop in behavior with the contralesional hand.

The ambidextrous monkey (case B) showed a similar overall drop in hand use, depending on the hand (Fig. $7 B$, Tables 2, 3 ), but was more pronounced compared with case $\mathrm{A}$. This may be a result of the delay in testing monkey A (72 vs $24 \mathrm{~h}$ after the lesion) or of a more distributed interhemispheric network for hand use in ambidextrous monkeys. Mean hand use in the analgesic control monkey remained the same throughout all phases of testing (Fig. 7C, Tables 2,3 ) ( $p>0.05$ between all phases).

For the lesioned monkeys, hand use returned to prelesion means by the fourth day postlesion. After recovery, the ambidextrous monkey remained ambidextrous, and the monkey with the left-hand preference maintained this left-hand preference. Our video analysis did not reveal any other effects on parameters such as accuracy, percentage of successful attempts, or retrieval success.

\section{Latencies}

Because of the low number of trials, statistical analysis for individual days after the lesion was not possible; however, there was a difference between prelesion means and the first three postlesion testing days when data were collapsed across days and wells. Thus, data from postlesion days 3, 4, and 5, when considered together, included significantly longer latencies than prelesion means collapsed across well locations and visual conditions. More specifically, in the visual condition with the left hand and nonvisual conditions with either hand for monkey A were significantly different when prelesion and postlesion latencies were compared $(p<0.5)$ (Tables 2, 3). A similar although nonsignificant trend was noted for monkey B. In our analgesic control monkey, the latencies before, during, and after analgesia were significantly different when left and right visual and nonvisual conditions were grouped together ( $t$ test with Bonferroni correction, $p<0.05$ ) (Tables 2, 3), but comparison of individual hands and conditions did not reach significance. Together, this suggests that the differences in latency to perform task 1 observed after the lesion may in part be due to the analgesics; however, the ability to perform and complete the task was not affected by the presence of analgesics.
Task 2: Time to Initiate
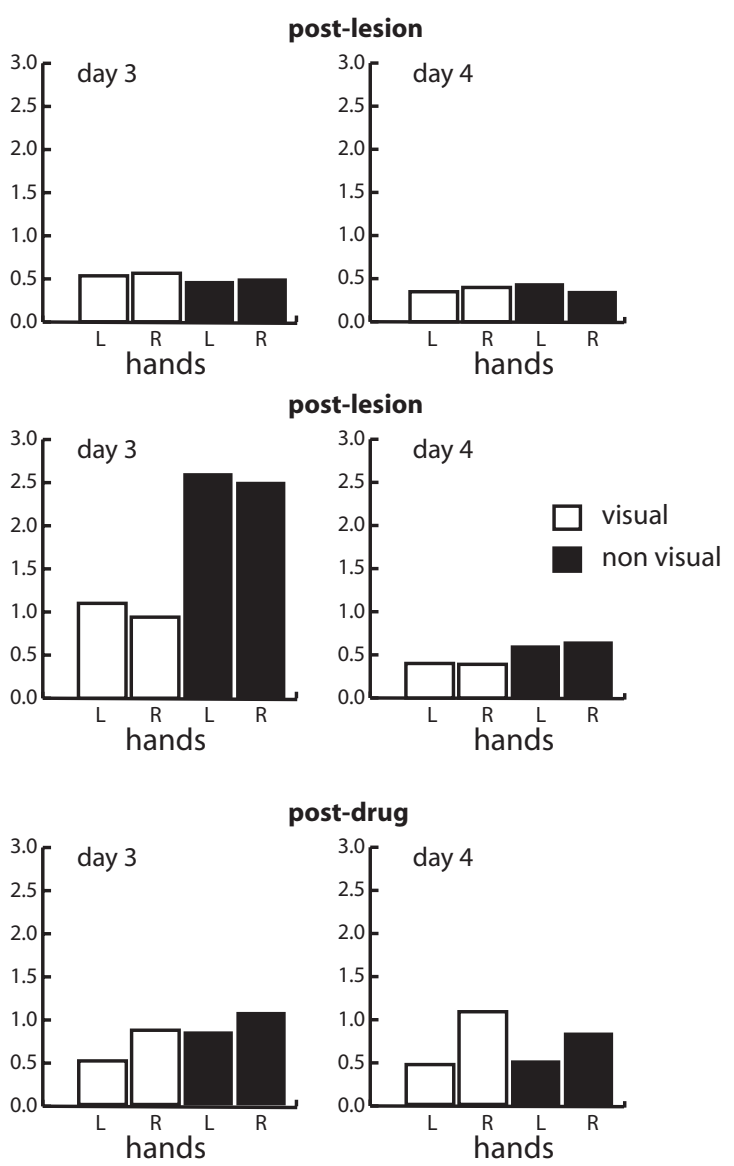

Fure 10. $\quad \boldsymbol{A}, \boldsymbol{B}$, Time to initiate task 2. For both lesion monkeys [case $A(\boldsymbol{A})$ and case $B(\boldsymbol{B})$ ], the time to initiate task 2 increased left and center conditions $(p<0.05)$ but returned to normal after the administration of analgesic. The dashed line marks the time of lesion, prelesion means were derived from session performed 1-10 d before the lesions were made. L, Left hand; $R$, right hand.

Task 2: visually and non-visually guided bimanual coordination Prelesion behavior for all monkeys during task 2 was highly consistent between monkeys, and between the nonvisual and visual conditions (Figs. 8, 9). Before the lesion, the monkeys always attempted to retrieve the reward from the correct location on their first attempt. All had close to $100 \%$ success at all three locations (Fig. 9A-C) and used a precision grip when retrieving the treat (Fig. 9D-F).

\section{Hand use}

In the visual condition, all monkeys had $100 \%$ or close to $100 \%$ use of the left hand when the left hand was required for retrieval before the lesion or administration of analgesic (Fig. $8 \mathrm{~A}-\mathrm{C}$ ). Performance was more variable in the nonvisual condition. Monkey A had a mean of 1.6 attempts at pellet retrieval from the center and right positions; monkey $\mathrm{B}$ retrieved the reward with the left hand in the first attempt in all positions, and the control monkey $\mathrm{AC}$ had a mean of 2.7 attempts in the center position (Fig. $8 D-\mathrm{F}$ ).

The largest effect of the area 5 lesions was in performance. The lesioned animals were unable to perform the task at the same rate and using the same strategies compared with their prelesion performance and with the analgesic control, and compared with their performance on the tactile discrimination task (Figs. 8, 9). The number of trials completed postlesion for task 2 was reduced from an average of 90 trials per day to 14 trials per day for case A, 


\section{Task 2: Overall Latency}

A Visual

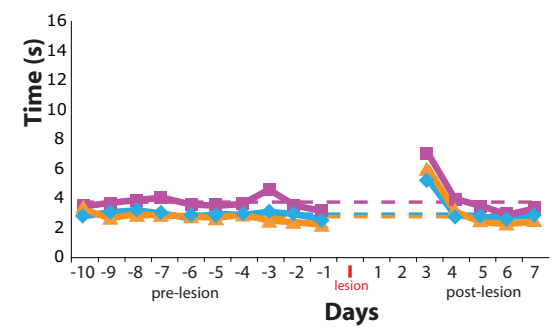

\section{Visual}

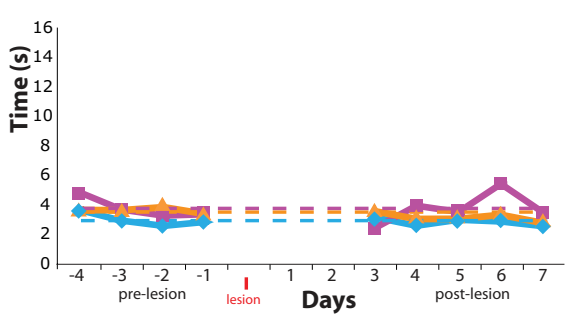

\section{E Visual}

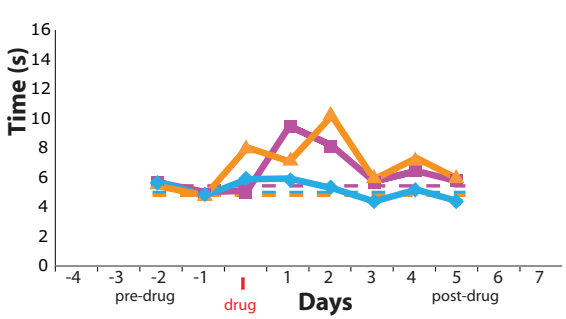

B Non-visual
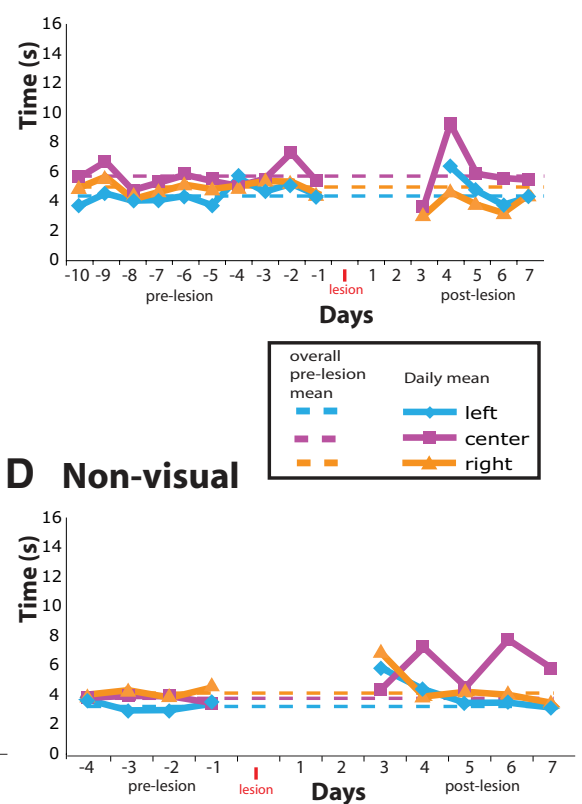

F Non-visual

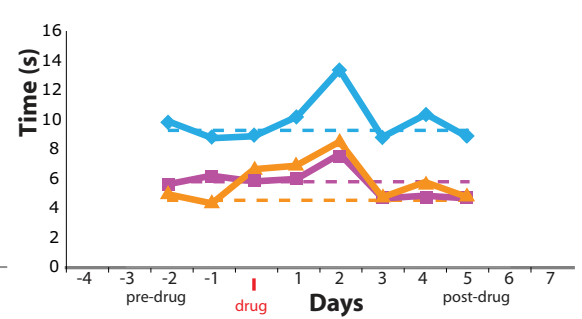

Figure 11. Overall latencies for task 2 . The latency to complete task 2 after the lesion was compared with prelesion means for both monkeys, and before, during, and after analgesic adiministration in the control monkey $(\boldsymbol{E}, \boldsymbol{F})$. $\boldsymbol{A}, \boldsymbol{B}$, For case $A$, the overall latency to complete task 2 was dramatically increased for all three well positions on postlesion day 3 , but returned to normal for all three well positions by postlesion day 4 for the visual condition. The latencies in the nonvisual condition were below prelesion means because the monkey did not perform the task or dropped the cylinder before the task was complete. The second monkey (case $B ; C, D$ ) only the nonvisual condition was affected such that latencies for the left and right location increased. The performance on the center location was erratic until the eighth postlesion day. $\boldsymbol{E}, \boldsymbol{F}$, For the analgesic control monkey, the latencies during administration of analgesics increased during the first day only, and the presence of the analgesic (oxymorphone) did not impair the animals ability to actually perform the task.

and from 91.3 to 26 trials a day for case B (on postlesion day 3 ). There was no drop in the number of trials for case AC thoughout the administration of oxymorphone.

The first lesioned monkey (case A) showed deficits primarily in the use of the contralesional hand. This left-handed animal showed a decrease in the percentage of times the left (contralesional) hand was used to retrieve the reward from the left or center position, a related decrease in the success of obtaining the reward for the left and center position, and an inability to use a precision grip when the reward was in the left or center position (Figs. $8 A, D, 9 A, D)$. This monkey had other deficits in the nonvisual condition (Figs. 8,9 ), such as a failure to use the left hand when the reward was in the left well position. He performed normally with the other hand when the reward was in the right or center position. All of these deficits recovered by either the fourth or fifth postlesion day.

The ambidextrous monkey (case B) also showed deficits in use of the left hand after the lesion, particularly at targets located in the center location, immediately in front of the animal. This was expressed as a decrease in the percentage of times the contralesional (left) hand was used to retrieve the reward from the center position, a decrease in the success of obtaining the reward from the center position, an inability to use a precision grip when the reward was in the center position, and an increase in the number of attempts required to retrieve the reward from the right location in the nonvisual condition (Figs. 8B,E, 9B,E). These deficits all recovered by the fifth postlesion day.

The results for the two lesioned monkeys in the immediate postlesion period stand in sharp contrast to those for the analgesic control monkey (Figs. 8C,F, 9C,F), whose hand use remained consistent across all of the parameters examined throughout the drug and postdrug periods.

\section{Latencies}

The mean time to initiate the task in both visual and nonvisual conditions ranged from 0.27 to $0.88 \mathrm{~s}$ before the lesion or the administration of analgesics (Fig. 10). After the lesion, the time to initiate the task increased modestly but not significantly in case $\mathrm{A}$ and in the analgesic control for most conditions (Fig. 10). In case B, much larger changes were observed, with an $89.7 \%$ increase in latency observed for left-handed visual trials, an increase of $36.2 \%$ for visual right-handed trials, an increase of $738.7 \%$ for nonvisual lefthanded trials, and an increase of 557.9\% for nonvisual right-handed trials in the third postlesion day. These differences appear large, but tests of statistical significance were not performed because of the low number of trials. Because we observed a significant increase in latency to initiate for the analgesic control in two nonvisual conditions - target presented to the right, and target centered (unpaired $t$ tests with Bonferroni correction; $p<0.05$ ) - it is likely that the increased reaction time for our lesioned monkeys on the third postlesion day (while analgesics were still being administered) was a result, in part, of the analgesics.

Likewise, the prelesion mean time to complete the task was similar for monkeys A and B at all well locations in the visual and nonvisual conditions (Fig. 11). Postlesion, overall latency to complete the task was also altered in all cases. But again, because the analgesic control also exhibited a significant increase in mean latency to complete task 2 (unpaired $t$ test, $p<0.01$ ) (Tables 2, 3), the latency increase on postlesion day 3 is likely attributable at least in part to the analgesics that were administered on that day.

Together, the behavioral results yield several consistent findings. First, after the lesion both monkeys used either the contralesional hand or both hands less for grasping, and performed many fewer trials on both tasks compared with prelesion hand use and performance, compared with the analgesic control and with the tactile discrimination task. Second, both monkeys had altered contralesional hand posture when entering the apparatus, and in 
using the precision grip when retrieving a reward at certain locations. Third, each monkey had a consistent deficit when performing the second, more difficult task 2, requiring the simultaneous use of both hands, but not the most difficult tactile discrimination task. Finally, the deficits that were observed recovered very rapidly, within $4-10 \mathrm{~d}$ after the lesion.

\section{Functional reorganization of areas 1 and} 2 after lesions to area 5

Cortical maps and receptive fields at selected recording sites made before the lesion were compared with immediate postlesion maps and receptive fields in one case, and with long-term maps and receptive fields $62-70 \mathrm{~d}$ after the lesion in both cases (Figs. 12-16). In case A, in which recordings were obtained immediately after the lesion, we observed substantial changes in the location and size of most recorded receptive fields (supplemental Fig. 1, available at www.jneurosci. org as supplemental material). For example, at some sites neurons that were previously responsive to stimulation of the wrist became responsive to stimulation of the glabrous palm after the lesion. At other sites, receptive fields moved from the digits to the thenar pad after the lesion. At the remaining sites, the receptive fields for neurons either remained the same, or changed in size from one digit to several digits or from all of the digits to portions of the same digits. The overlap index for the seven sites in which prelesion and postlesion receptive fields were obtained was $16.8 \%$. Examination of the receptive fields in supplemental Figure 1 (available at www.jneurosci.org as supplemental material) reveals that all but one comparison, receptive field I, had $0 \%$ or low overlap indices.

In both monkeys, cortex was explored extensively $62 \mathrm{~d}$ (case B) or $70 \mathrm{~d}$ (case A) after the lesion. Electrophysiological recording sites were matched across time points, as described in Materials and Methods and illustrated in Figure 5. Our goal was to compare recording sites that were closely situated to each other across time points. Over the 2 month period after the lesion, receptive fields for neurons in much of the hand representation of areas 2 and 1 changed significantly (Figs. 13, 14) compared with prelesion receptive fields. This was accompanied by very large overall changes in the functional organization of areas 2 and 1 in that the representation of the glabrous digits and palm shrank in size compared with prelesion maps and maps of normal animals (Figs. 13, 14, 16).

In case $\mathrm{A}, 70 \mathrm{~d}$ after the lesion, receptive fields for neurons in areas 1 and 2 that were in matched locations in cortex in most instances changed location on the skin and body $70 \mathrm{~d}$ after the lesion (Fig. 13). For example, in area 2, neurons that previously had receptive fields on the digits now had receptive fields on the glabrous pads (Fig. 13, RFs 3 and 4), on the thenar pad, or on the dorsal digits. Two of these sites (sites 3 and 4) were recorded immediately after the lesion, and the receptive fields at that time

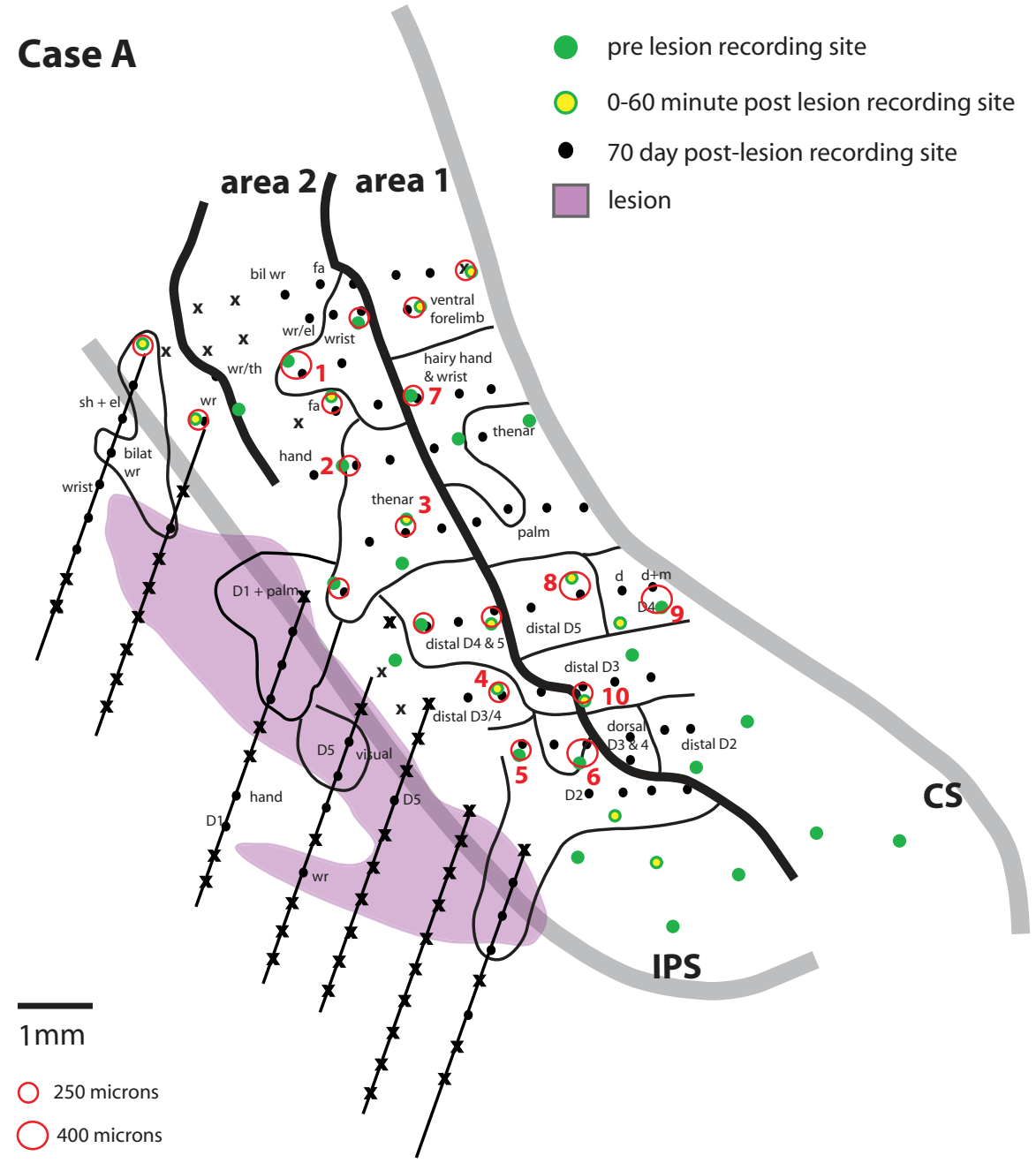

Figure 12. A comprehensive reconstruction in which prelesion, immediate postlesion, and $70 \mathrm{~d}$ postlesion recording sites have been merged (case A). Some sites from the different maps are identical and others are within a 250-400 $\mu \mathrm{m}$ diameter from each other. The circles at the bottom indicate scaled diameters for recording site comparison. Sites that were farther apart were not considered for is comprehensive map match those on the map in Fig. 15. Abbreviations are defined in Table 1.

point differed from the original receptive field, and at one site (site 4) differed from the receptive field observed $70 \mathrm{~d}$ later (site 4). In area 1 , neurons at some sites had changes in location and size of receptive fields after the lesion. For example, at one site (site 7) neurons had a relatively small receptive field on the wrist before the lesion and a larger receptive field that extended onto the distal forelimb $70 \mathrm{~d}$ after the lesion. At another site (site 9), neurons had a small receptive field on the proximal portion of glabrous digit 4 . Seventy days after the lesion, the receptive field shifted to the distal portion of digit 4. Calculation of the overlap index for all sites evaluated indicates that for areas 1 and 2 there is only a $20 \%$ overlap in receptive fields before and $70 \mathrm{~d}$ after the lesion. The amount of cortex that represented the glabrous hand and digits was also calculated before the lesion and $70 \mathrm{~d}$ after the lesion. Before the lesion, the glabrous hand and digits occupied $29.8 \mathrm{~mm}^{2}$ of area 1 and $46.5 \mathrm{~mm}^{2}$ of area 2, for a total of 76.3 $\mathrm{mm}^{2}$. Seventy days after the lesion, the amount of cortex representing the glabrous hand and palm in areas 1 and 2 dropped to 18.5 and $16.8 \mathrm{~mm}$, respectively (total, $-35.3 \mathrm{~mm}^{2}$ ).

In the second case (case B) in which prelesion and long-term postlesion maps and receptive fields were compared (Fig. 14; supplemental Fig. 2, available at www.jneurosci.org as supplemental material), we observed similar changes in overall map 


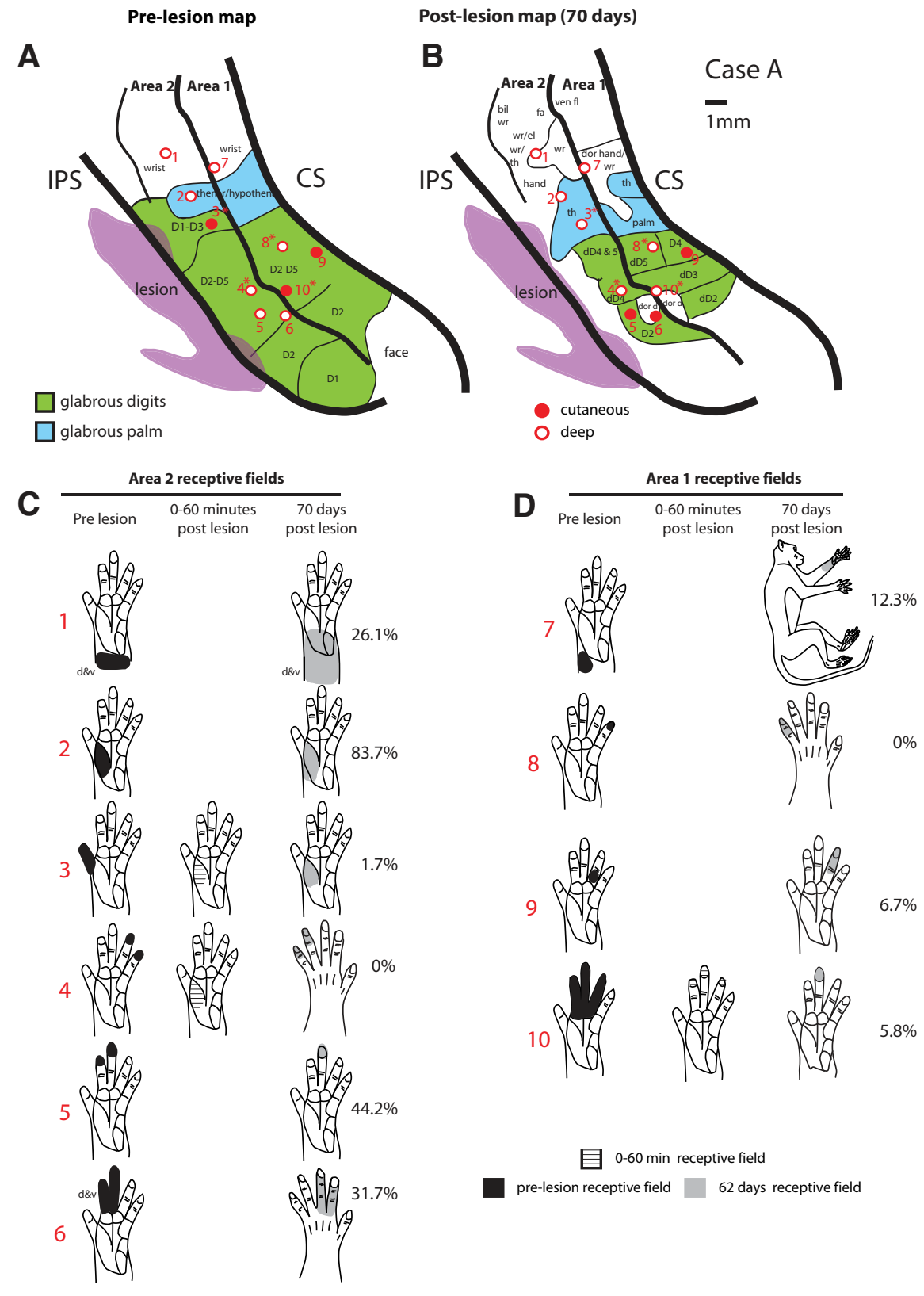

Figure 13. $\boldsymbol{A}-\boldsymbol{D}$, Reconstructions of a prelesion map $(\boldsymbol{A})$, a $70 \mathrm{~d}$ postlesion map $(\boldsymbol{B})$, and corresponding receptive fields for neurons in area $2(\boldsymbol{C})$ and area $1(\boldsymbol{D})$ in case $A$. $\boldsymbol{A}, \boldsymbol{B}$, Representations of the glabrous hand are shaded in green and representations of the glabrous palm are shaded in blue. Representations of the glabrous hand decreased in size after the lesion and receptive fields for neurons at most sites changed locations $70 \mathrm{~d}$ after the lesion, and in most instances immediately after the lesion (e.g., RF 3, 4 and 10). The overall overlap index for this monkey was $20 \%$. Conventions are as in Figure 12 . Abbreviations are as defined in Table 1.

organization with a large reduction in the representation of the glabrous digits and palm, and an alteration in receptive field location (Fig. 14A, B). As in the other case, the amount of cortex devoted to the glabrous hand and palm representation in area 1 was reduced from $29 \mathrm{~mm}^{2}$ prelesion to $12.2 \mathrm{~mm}^{2}$ postlesion.

\section{Normal variability in receptive field size and location in areas 1 and 2}

To establish that the cortical plasticity that we observed after lesions to area 5 was attributable to the lesion, and not the natural plasticity mechanisms inherent in the neocortex of all mammals or discrepancies in recording site location at different time points, we assessed data from one normal monkey that had been examined as part of an unrelated study at two time points separated by $10 \mathrm{~d}$. Electrophysiological recordings were made at several locations in the representation of the digits and palm of areas 1 and 2, receptive fields were determined, and then cortex was remapped $10 \mathrm{~d}$ later (Fig. 15). We observed that receptive fields for neurons remain relatively constant over time (Fig. 15 , RFs 2, 4, and 7) with slight shifts in location on the digit (Fig. 15, RF 8) or palm (RF 11), or in the size of receptive fields (Fig. 15, RF 1). The overlap index was $43 \%$, over twice that of monkey A (20\%) and B (0\%). In instances where the overlap index was low, the receptive field was very small but was still located very close to the original receptive field (RFs 8 and 11) or the placement of the electrode site was at the conjunction of several body part representations (e.g., RF 10). Thus, although there are slight changes in the location of receptive fields for neurons across time points within a cortical field, at most sites receptive field location remained constant when compared with our lesioned animals.

We also compared our postlesion maps with maps of area 2 and 1 in two normal monkeys from our own laboratory in which identical recording techniques were used, and maps from the study of Pons et al. (1985) in which similar, although not identical, recording techniques were used. We found that there was variability in the organization of areas 1 and 2 in the three monkeys examined (Fig. 16A-C). However, the overall size of the glabrous hand representation in all three animals was consistent, and representations of all of the glabrous digits could be observed. In the Pons et al. (1985) study, the overall size of the glabrous hand representation in areas 1 and 2 was $46.7 \mathrm{~mm}^{2}$ (Fig. 16A). In the two normal animals from our own laboratory, the overall size of the glabrous hand representation in areas 1 and 2 in the second case (Fig. 16B, C) averaged $48.7 \mathrm{~mm}^{2}$ (47.39 and $51.97 \mathrm{~mm}^{2}$, respectively). This is in striking contrast to the size of the glabrous digit and palm representation in areas 1 and 2 in the lesioned monkey $\mathrm{A}\left(35.3 \mathrm{~mm}^{2}\right)$ and $\mathrm{B}(23$ $\mathrm{mm}^{2}$ ) (Fig. 16D). Together, the data indicate two major alterations in areas 1 and 2 after lesions to area 5. First, the overall topography of the map changed in that the glabrous digits and palm representation decreased in size compared with earlier maps of these fields in the same animal (Figs. 13A, B, 14A,B) and compared with maps of areas 1 and 2 in normal animals (Fig. 16). Second, the receptive field location changed immediately after the lesion (1-60 $\mathrm{min}$ ) as well as 2 months after the lesion. These dramatic changes in receptive field location are not observed in normal animals in which receptive field location was assessed at a 10 day interval (Fig. 15). 


\section{Discussion}

In the present study, focal lesions to posterior parietal area 5 resulted in several deficits in reaching and grasping that recovered quite rapidly. This recovery was accompanied by a reorganization of somatosensory areas 1 and 2 . This reorganization may simply be a result of diaschisis, retrograde changes from loss of area 5 . Although these changes in cortical organization are correlative, it is possible that they may contribute in part to the behavioral recovery observed after lesions of area 5 .

Previous studies demonstrate that area 5 is involved in coordinating or programming intention of movement (Snyder et al., 1997; Debowy et al., 2001; Krubitzer and Disbrow, 2008 for review), and generating appropriate coordinates for reaching (Ferraina and Bianchi, 1994; Lacquaniti et al., 1995) and grasping (Gardner et al., 2007a,b). Metabolic studies demonstrate that activity in area 5 (and anterior parietal areas) increases during reaching, even when that hemisphere is deprived of visual input (Savaki et al., 1993), and that area 5 is particularly important for reaching in nonvisual conditions (Gregoriou and Savaki, 2001).

Given the importance of area 5 for reaching and grasping, it is surprising that the behavioral deficits that we observed after lesions to the area were subtle and short lived. Our most profound results were a considerable decrease in hand use after our lesions, an apparent neglect of a specific portion of task space (e.g., contralesional or midline), and an inability to use preferred hand posture. The deficits were most marked in the task that required simultaneous use of both hands. While our analgesic control indicates that task latency may in fact be attributable to the presence of analgesics, they do not account for the severely reduced ability to perform either task or the altered performance level and strategy. Further, the ability to use the ipsilesional hand relatively normally, and the normal performance on the more difficult nonreaching, nonvisual texture discrimination task indicates that general cognitive and motor function was unaffected by the surgery during the time of postlesion testing.

Previous studies in which area 5 was lesioned together with other areas such as medial intraparietal sulcus (MIP) and area 7b (Rushworth et al., 1997a,b, 1998) in macaque monkeys demonstrated an inability to coordinate arm velocity, a disruption of the postural relationship between the arm and wrist, and disruptions in coordinating the hand in shoulder-centered space. Studies in which larger portions of posterior parietal cortex was lesioned [including at least areas 5 and 7, PRR, AIP, vascular intraparietal area, MIP, and lateral intraparietal are (LIP)] demonstrate that monkeys have a reluctance to use the contralesional hand immediately after the lesion, as in the present study (Lamotte and Acuña,
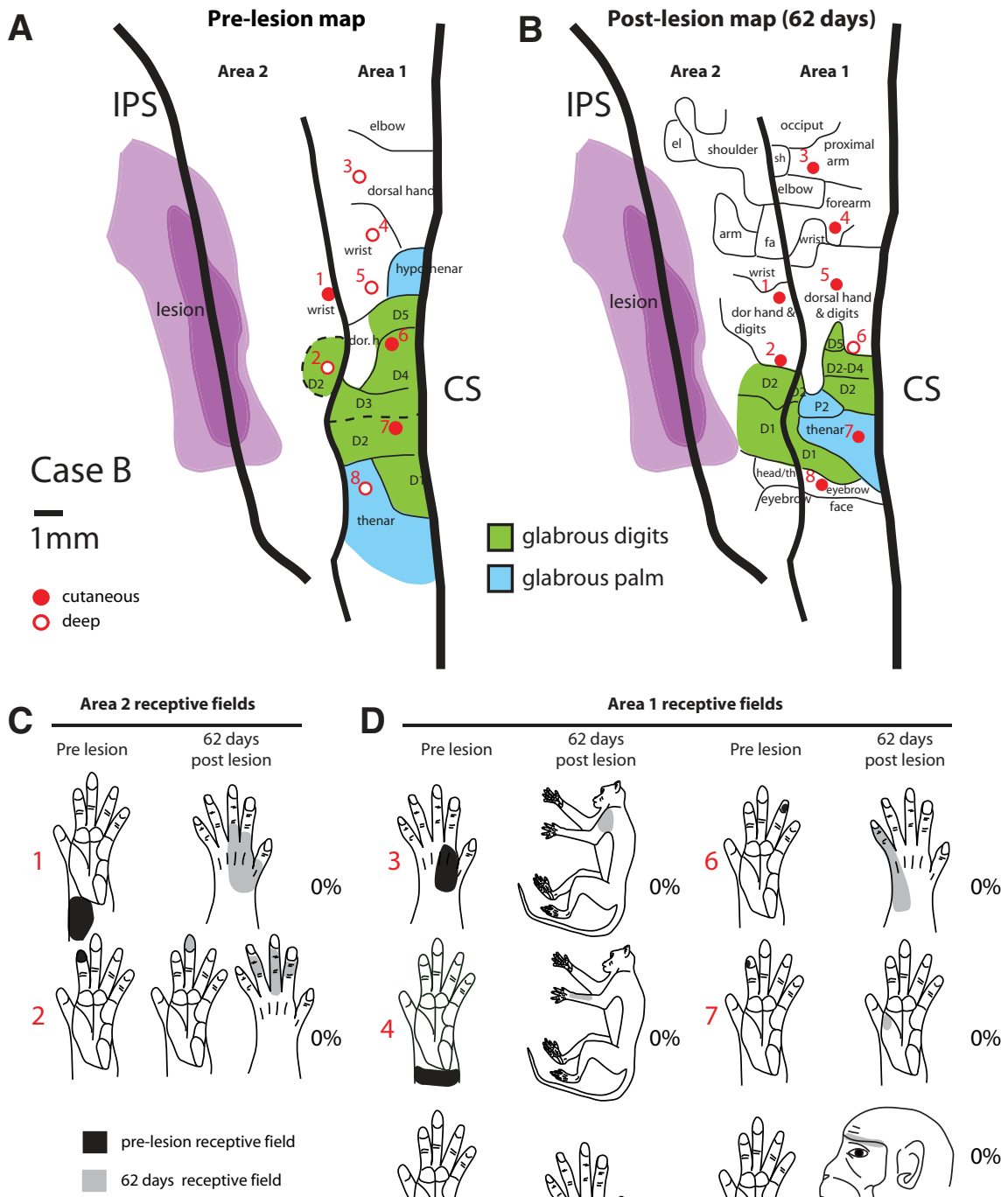

$0 \%$
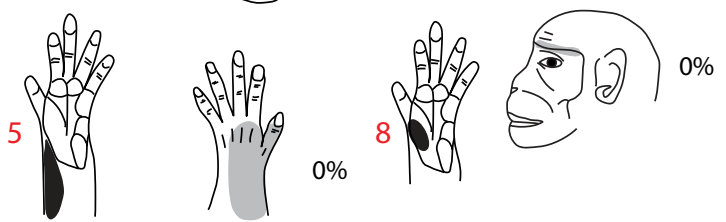

$0 \%$

Figure 14. $A-D$, Reconstructions of a prelesion map $(\boldsymbol{A})$ and a $62 \mathrm{~d}$ postlesion map $(\boldsymbol{B})$, and corresponding receptive fields for neurons in area $2(\boldsymbol{C})$ and area 1 (D) in case $B . \boldsymbol{A}, \boldsymbol{B}$, Representations of the glabrous hand are shaded in green and representations of the glabrous palm are shaded in blue. The representation of the glabrous hand in area 1 decreased in size after the lesion, and receptive fields for neurons at most sites changed locations $62 \mathrm{~d}$ after the lesion. The overall overlap index in this case was $0 \%$. Conventions are as in previous figures. Abbreviations are as defined in Table 1.

1978). Further, these very large ablations result in severe and persistent deficits in reaching in visual space, use of improper orientations of the hand while reaching, and an increase in the direction of the reaching error when visual information was absent.

As in the present investigation, animals that had relatively smaller lesions (e.g., areas 5 and $7 \mathrm{~b}$, and AIP) (Rushworth et al., 1997a,b, 1998) recovered manual abilities very rapidly, indicating that spared regions of cortex can compensate for the lost region within days of the loss. However, when very large regions of cortex are lesioned (e.g., Lamotte and Acuña, 1978), this compensatory ability is greatly diminished.

\section{Cortical plasticity}

A number of previous studies demonstrate that the functional organization of cortical fields can be altered by changes in peripheral inputs and the acquisition of new skills, which lead to expansions and contractions of cortical maps, receptive fields, and 
A Normal Monkey

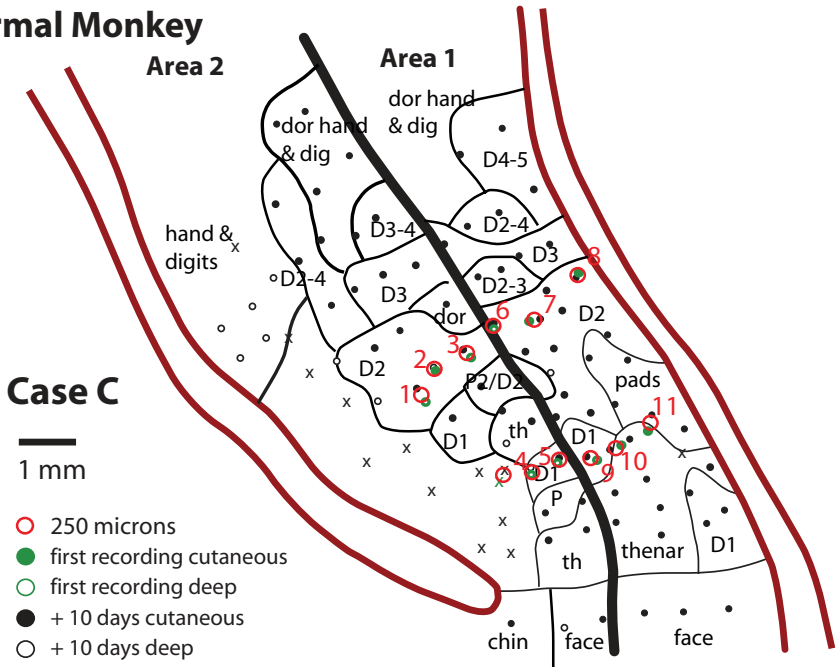

B Area 2 receptive fields

first recordings 10 days later

C

Area 1 receptive fields
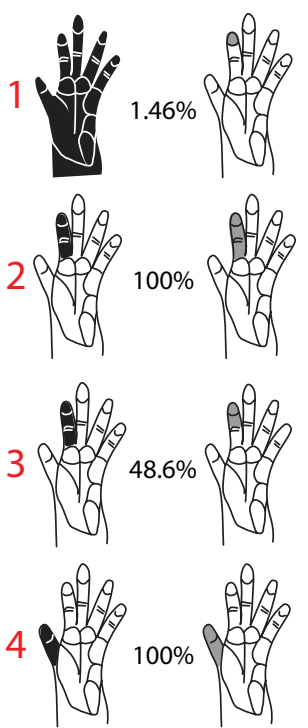

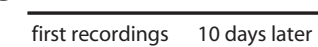

first recordings 10 days later
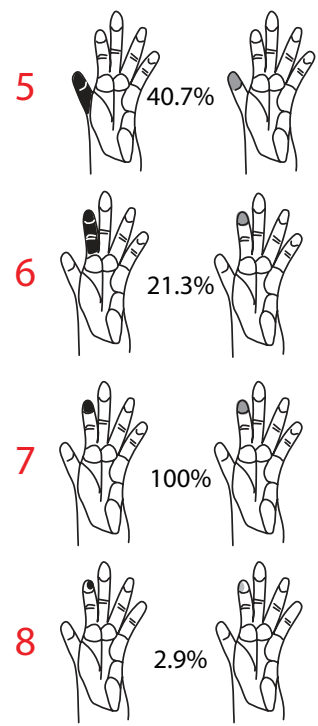
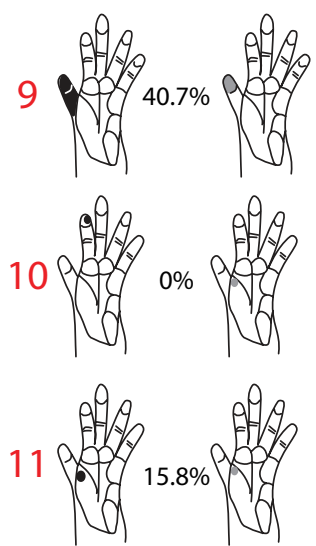

first receptive field

+10 days receptive field

Figure 15. $\quad \boldsymbol{A}-\boldsymbol{C}$, Maps of areas 1 and $2(\boldsymbol{A})$ with corresponding receptive fields at marked locations in areas $2(\boldsymbol{B})$ and $1(\boldsymbol{C})$ in a normal monkey. This map was generated from multiple recording sites in case C. At recording sites 1-11, receptive fields were obtained at 2 time points. An early time point (black receptive fields) and $10 \mathrm{~d}$ later (gray receptive fields). The location of the receptive fields was very similar across both time points except at two locations. The overall overlap index for this case was $43 \%$. Abbreviations are as defined in Table 1. Conventions are as in previous figures.

changes in temporal characteristics of neurons (Merzenich et al., 1984; Recanzone et al., 1992a,b; Nudo, 1999; Lissek et al., 2009; but, see also Darian-Smith, 2008). Fewer studies have examined the reorganization of somatosensory cortex after cortical lesions. Pons et al. $(1987,1988,1992)$ demonstrated that lesions to the hand representation in S1 of monkeys result in an immediate expansion of the hand representation in S2. Xerri et al. (1998) demonstrate that focal lesions to area $3 \mathrm{~b}$ in owl monkeys result in the emergence of new cutaneous representations of the digits in adjacent areas 1 and 2, particularly for the distal digit tips of D3 and D4. This emergence was accompanied by the reacquisition of a retrieval skill requiring the use of these distal digits. Studies of motor cortical lesions in squirrel monkeys demonstrate that surrounding the lesion, motor representations of a particular body part may contract or expand, and skill reacquisition is accompanied by predictable reorganization of the spared cortex (Nudo et al., 1996; Nudo and Milliken, 1996; Dancause et al., 2006; Friel et al., 2007).

One surprising result from the present study was that the glabrous hand representation in areas 1 and 2 decreased dramatically after these lesions, compared with prelesion maps, and these alterations far exceeded the variability observed in normal animals. Although these results are intriguing, it is important to discuss several considerations. First, it is possible that the map changes that we observed in our lesioned monkeys compared with normal monkeys are the result of extensive training of our monkeys on several manual tasks. Second, our normal control was mapped $10 \mathrm{~d}$ apart, whereas maps of our lesioned monkeys were obtained 62 and $70 \mathrm{~d}$ after the initial mapping. Although it is possible that hand use played a role in the reorganization of areas 1 and 2 that we observed, two considerations make this unlikely. Previous studies (Jenkins et al., 1990; Recanzone et al., 1992a,b, 1993; Wang et al., 1995; Xerri et al., 1998, 1999) demonstrate that extended use or skilled learning actually expands the sensory representation that was trained rather than diminishing it. In addition, the animals were extensively trained before the lesion, and any use-dependent cortical changes would be expected to have occurred and solidified before the lesion was induced. The second issue is in the comparison in timing between maps in lesioned and control animals. In one lesioned animal, recordings were made within $60 \mathrm{~min}$ after the lesion, and the alterations in receptive fields were already present. Further, the overlap of receptive fields was much greater in normal animals (overlap index, 43\%) with small location shifts (e.g., ulnar vs radial sides of D1 tip) compared with lesioned animals ( 0 and 20\%), which had large location shifts (hand vs eyebrow; hand vs shoulder). Finally, the difference in the glabrous representation of the postlesion maps compared with several maps from unlesioned animals (including the prelesion case) were vastly different, which could not be accounted for by individual idiosyncrasies. Thus, the immediate and long-term changes observed in the receptive fields and the related map reorganization are likely the result of the area 5 lesion rather than normal variability or skilled use.

We suggest that these alterations play a role in the behavioral recovery that we observed; however, it is not immediately clear how a reduction in the glabrous hand representation in these fields relates to behavioral recovery. Further, one would expect that the reduction in the glabrous hand representation in areas 1 and 2 would negatively affect the tactile discrimination task. First, it is possible that other areas such as the AIP in conjunction with areas 1 and 2 compensate for area 5 and contribute to the recovery of behavior, since many of the neurons in AIP are active during similar portions of a manual 
tasks as neurons in area 5 (Gardner et al., 2007a,b). Second, it is likely that area $3 b$ could mediate the discriminations necessary to perform the tactile discrimination task. An important implication of these somewhat counterintuitive results is that loss of one node in a processing network (area 5) clearly affects the network as a whole, and that subtle alterations in cortical representations and neuronal responses within spared fields can compensate for focal disruption to a portion of the network. However, the extent of circuit modification, and ultimately how and when functional changes are consolidated, requires further investigation.

\section{Neural substrate of rapid cortical plasticity}

The rapidity of the cortical plasticity described here $(60 \mathrm{~min}$ to $60 \mathrm{~d}$ ) is consistent with a change in the functional properties of existing anatomical connections. Neuroanatomical studies in macaques provide evidence that AIP and area 2 have some of the same cortical inputs as area 5, particularly with motor and premotor cortex, and areas on the caudal bank of the IPS such as LIP (Pons and Kaas, 1986; Disbrow et al., 2000; Nakamura et al., 2001; Borra et al., 2008). Similarly, there is a high degree of overlap of the thalamocortical projections to anterior and posterior parietal areas. Recent studies in which topographically matched representations in areas $3 a, 3 b, 1,2$, and 5 were injected with anatomical tracers reveal a tremendous amount of divergence and convergence in thalamocortical connections (Padberg et al., 2009). Each thalamic nucleus projects to several cortical fields, and each cortical field receives input from multiple thalamic nuclei. Thus, the thalamocortical and corticocortical afferents already present can provide the scaffolding for the reorganization of areas 2 and 1 , and the accompanying behavioral recovery that we observe.

\section{References}

Borra E, Belmalih A, Calzavara R, Gerbella M, Murata A, Rozzi S, Luppino G (2008) Cortical connections of the macaque anterior intraparietal (AIP) area. Cereb Cortex 18:1094-1111.

Culham JC, Danckert SL, DeSouza JF, Gati JS, Menon RS, Goodale MA (2003) Visually guided grasping produces fMRI activation in dorsal but not ventral stream brain areas. Exp Brain Res 153:180-189.

Culham JC, Cavina-Pratesi C, Singhal A (2006) The role of parietal cortex in visuomotor control: what have we learned from neuroimaging? Neuropsychologia 44:2668-2684.

Dancause N, Barbay S, Frost SB, Zoubina EV, Plautz EJ, Mahnken JD, Nudo RJ (2006) Effects of small ischemic lesions in the primary motor cortex on neurophysiological organization in ventral premotor cortex. J Neurophysiol 96:3506-3511.
A Pons et al., 1985 - Normal

\section{B Normal: Case C}

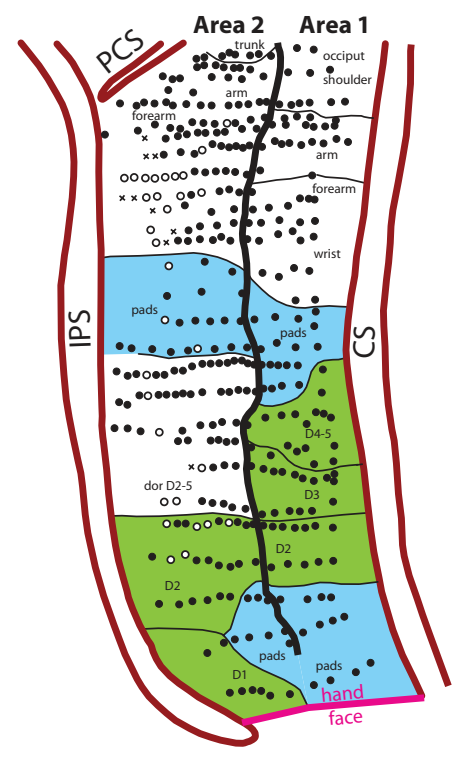

C Normal: Case D
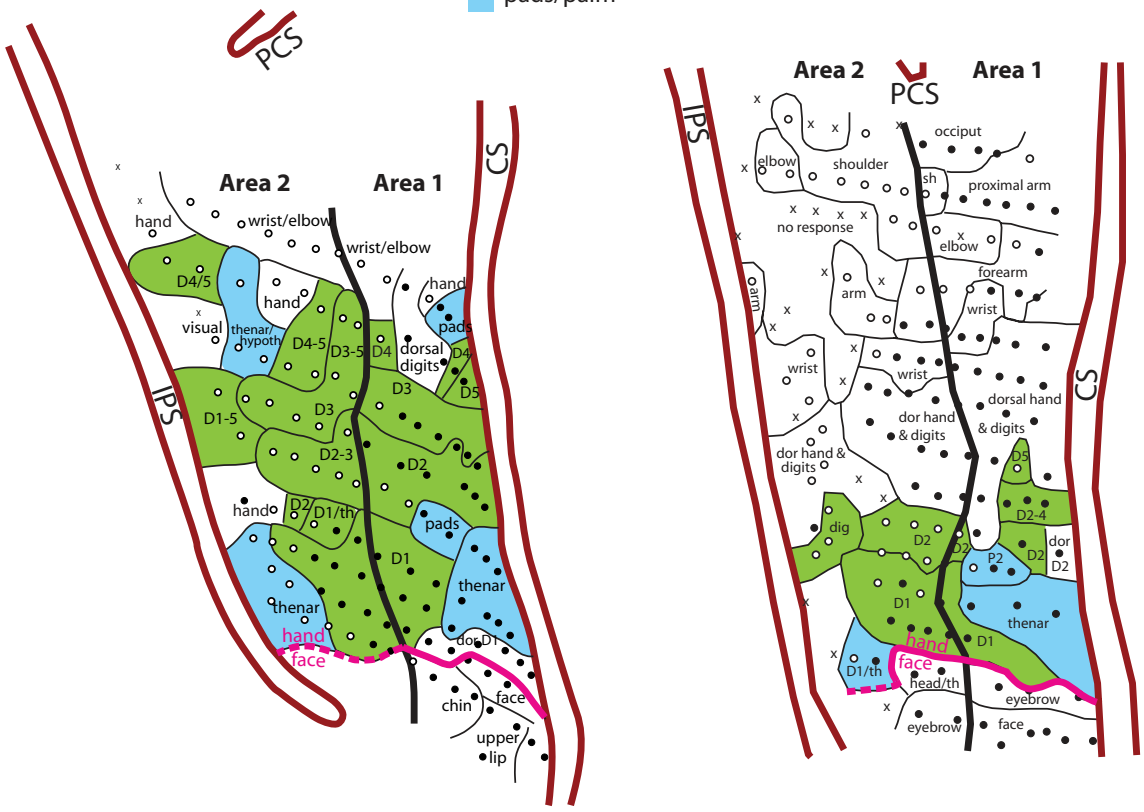

Figure 16. $\quad \boldsymbol{A}-\boldsymbol{D}$, Maps of areas 1 and 2 in three normal monkeys $(\boldsymbol{A}-\boldsymbol{C})$, and in one monkey in which area 5 was lesioned $(\boldsymbol{D})$. The map in $\boldsymbol{A}$ was generated by Pons et al. (1985), and the maps in $\boldsymbol{B}$ and $\boldsymbol{C}$ were generated in our own laboratory. Representations of the glabrous digits are shaded in green and representations of the glabrous palm is marked in blue. The hand and face boundary is marked as a pink line. While there is variability in the organization of areas 1 and 2 in normal monkeys, the representation of the glabrous hand is quit large in these monkeys, and representations of all digits are observed. The area of cortex devoted to the representation of the glabrous digits and palm in areas 1 and 2 in these monkeys is $46.7 \mathrm{~mm}^{2}$ in the Pons et al. (1985) study, $47.39 \mathrm{~mm}^{2}$ in case C, and $23 \mathrm{~mm}^{2}$ in the lesioned monkey $(\boldsymbol{D})$. The size of the glabrous hand representation in in the lesioned monkey is well outside the normal variability observed in normal monkeys. Abbreviations are as defined in Table 1. Conventions as are in previous figures.

Darian-Smith C (2008) Plasticity of somatosensory function during learning, disease and injury. In: The senses: a comprehensive reference (Kaas J, Gardner E, eds), pp 259-297. London: Elsevier.

Debowy DJ, Ghosh S, Ro JY, Gardner EP (2001) Comparison on neuronal firing rates in somatosensory and posterior parietal cortex during prehension. Exp Brain Res 137:269-291.

Disbrow E, Huffman K, Recanzone G, Krubitzer L (2000) The connections of areas 5 and 2 with electrophysiologically identified somato- 
sensory cortical areas in macaque monkeys. Soc Neurosci Abstr 26:2082.

Disbrow E, Hinkley L, Padberg J, Krubitzer L (2007) Hand use and the evolution of posterior parietal cortex in primates. In: Evolution of nervous systems (Kaas J, Preuss T, eds), pp 407-415. London: Elsevier.

Ferraina S, Bianchi L (1994) Posterior parietal cortex: Functional properties of neurons in area 5 during an instructed-delay reaching task within different parts of space. Exp Brain Res 99:175-178.

Friel KM, Barbay S, Frost SB, Plautz EJ, Stowe AM, Dancause N, Zoubina EV, Nudo RJ (2007) Effects of a rostral motor cortex lesion on primary motor cortex hand representation topography in primates. Neurorehabil Neural Repair 21:51-61.

Gallyas F (1979) Silver staining of myelin by means of physical development. Neurol Res 1:203-209.

Gardner E (2008) Dorsal and ventral streams in the sense of touch. In: The senses: a comprehensive reference (Kaas J, Gardner E, eds), pp 233-258. London: Elsevier.

Gardner EP, Babu KS, Reitzen SD, Ghosh S, Brown AS, Chen J, Hall AL, Herzlinger MD, Kohlenstein JB, Ro JY (2007a) Neurophysiology of prehension. I. Posterior parietal cortex and object-oriented hand behaviors. J Neurophysiol 97:387-406.

Gardner EP, Babu KS, Ghosh S, Sherwood A, Chen J (2007b) Neurophysiology of prehension. III. Representation of object features in posterior parietal cortex of the macaque monkey. J Neurophysiol 98:3708-3730.

Gregoriou GG, Savaki HE (2001) The intraparietal cortex: subregions involved in fixation, saccades, and in the visual and somatosensory guidance of reaching. J Cereb Blood Flow Metab 21:671-682.

Hinkley LB, Krubitzer LA, Padberg J, Disbrow EA (2009) Visual-manual exploration and posterior parietal cortex in humans. J Neurophysiol 102:3433-3446.

Hörster W, Ettlinger G (1985) An association between hand preference and tactile discrimination performance in the rhesus monkey. Neuropsychologia 23:411-413.

Iwamura Y, Tanaka M, Iriki A, Taoka M, Toda T (2002) Processing of tactile and kinesthetic signals from bilateral sides of the body in the postcentral gyrus of awake monkeys. Behav Brain Res 135:185-190.

Jenkins WM, Merzenich MM, Ochs MT, Allard T, Guíc-Robles E (1990) Functional reorganization of primary somatosensory cortex in adult owl monkeys after behaviorally controlled tactile stimulation. J Neurophysiol 63:82-104.

Kalaska JF (1996) Parietal cortex area 5 and visuomotor behavior. Can J Physiol Pharmacol 74:483-498.

Krubitzer L, Disbrow E (2008) The evolution of parietal areas involved in hand use in primates. In: The senses: a comprehensive reference (Kaas J, Gardner E, eds), pp 183-214. London: Elsevier.

Lacquaniti F, Guigon E, Bianchi L, Ferraina S, Caminiti R (1995) Representing spatial information for limb movement: the role of area 5 in monkey. Cereb Cortex 5:391-409.

Lamotte RH, Acuña C (1978) Deficits in accuracy of reaching after removal of posterior parietal cortex in monkeys. Brain Res 139:309-326.

Lissek S, Wilimzig C, Stude P, Pleger B, Kalisch T, Maier C, Peters SA, Nicolas V, Tegenthoff M, Dinse HR (2009) Immobilization impairs tactile perception and shrinks somatosensory cortical maps. Curr Biol 19:837-842.

Merzenich MM, Kaas JH, Wall JT, Sur M, Nelson RJ, Felleman DJ (1983) Progression of change following median nerve section in the cortical representation of the hand in areas $3 \mathrm{~b}$ and 1 in adult owl and squirrel monkeys. Neuroscience 10:639-665.

Merzenich MM, Nelson RJ, Stryker MP, Cynader MS, Schoppmann A, Zook JM (1984) Somatosensory cortical map changes following digit amputation in adult monkeys. J Comp Neurol 224:591-605.

Mountcastle VB, Lynch JC, Georgopoulos A, Sakata H, Acuna C (1975) Posterior Parietal Association Cortex of the Monkey: Command Functions for Operations Within Extrapersonal Space. J Neurophysiol 38:871-908.

Nakamura H, Kuroda T, Wakita M, Kusunoki M, Kato A, Mikami A, Sakata H, Itoh K (2001) From three-dimensional space vision to prehensile hand movements: the lateral intraparietal area links the area V3A and the anterior intraparietal area in macaque monkeys. J Neurosci 21:8174-8187.

Nelson RJ, Sur M, Felleman DJ, Kaas JH (1980) Representations of the body surface in postcentral parietal cortex of Macaca fascicularis. J Comp Neurol 192:611-643.

Nudo RJ (1999) Recovery after damage to motor cortical areas. Curr Opin Neurobiol 9:740-747.
Nudo RJ, Milliken GW (1996) Reorganization of movement representations in primary motor cortex following focal ischemic infarcts in adult squirrel monkeys. J Neurophysiol 75:2144-2149.

Nudo RJ, Wise BM, SiFuentes F, Milliken GW (1996) Neural substrates for the effects of rehabilitative training on motor recovery after ischemic infarct. Science 272:1791-1794.

Padberg J, Disbrow E, Krubitzer L (2005) The organization and connections of anterior and posterior parietal cortex in titi monkeys: do New World monkeys have an area 2? Cereb Cortex 15:1938-1963.

Padberg J, Franca JG, Cooke DF, Soares JG, Rosa MG, Fiorani M Jr, Gattass R, Krubitzer L (2007) Parallel evolution of cortical areas involved in skilled hand use. J Neurosci 27:10106-10115.

Padberg J, Cerkevich C, Engle J, Rajan AT, Recanzone G, Kaas J, Krubitzer L (2009) Thalamocortical connections of parietal somatosensory cortical fields in macaque monkeys are highly divergent and convergent. Cereb Cortex 19:2038-2064.

Pons TP, Kaas JH (1986) Corticocortical connections of area 2 of somatosensory cortex in macaque monkeys: a correlative anatomical and electrophysiological study. J Comp Neurol 248:313-335.

Pons TP, Garraghty PE, Cusick CG, Kaas JH (1985) The somatotopic organization of area 2 in macaque monkeys. J Comp Neurol 241:445-466.

Pons TP, Garraghty PE, Mishkin M (1988) Lesion-induced plasticity in the second somatosensory cortex of adult macaques. Proc Natl Acad Sci U S A 85:5279-5281.

Pons TP, Garraghty PE, Mishkin M (1992) Serial and parallel processing of tactual information in somatosensory cortex of rhesus monkeys. J Neurophysiol 68:518-527.

Pons T, Garraghty PE, Mishkin M (1987) Topographical reorganization in the second somatosensory area (SII) after removal of selected portions of the postcentral cortex. Soc Neurosci Abstr 13:341.

Recanzone GH, Merzenich MM, Jenkins WM, Grajski KA, Dinse HR (1992a) Topographic reorganization of the hand representation in cortical area $3 \mathrm{~b}$ of owl monkeys trained in a frequency discrimination task. J Neurophysiol 67:1031-1056.

Recanzone GH, Merzenich MM, Jenkins WM (1992b) Frequency discrimination training engaging a restricted skin surface results in an emergence of a cutaneous response zone in cortical area 3a. J Neurophysiol 67:1057-1070.

Recanzone GH, Schreiner CE, Merzenich MM (1993) Plasticity in the frequency representation of primary auditory cortex following discrimination training in adult owl monkeys. J Neurosci 13:87-103.

Rushworth MF, Nixon PD, Passingham RE (1997a) Parietal cortex and movement. I. Movement selection and reaching. Exp Brain Res 117: 292-310.

Rushworth MF, Nixon PD, Passingham RE (1997b) Parietal cortex and movement. II. Spatial representation. Exp Brain Res 117:311-323.

Rushworth MF, Johansen-Berg H, Young SA (1998) Parietal cortex and spatial-postural transformation during arm movements. J Neurophysiol 79:478-482.

Savaki HE, Kennedy C, Sokoloff L, Mishkin M (1993) Visually guided reaching with the forelimb contralateral to a "blind" hemisphere: a metabolic mapping study in monkeys. J Neurosci 13:2772-2789.

Snyder LH, Batista AP, Andersen RA (1997) Coding of intention in the posterior parietal cortex. Nature 386:167-170.

Taoka M, Toda T, Iwamura Y (1998) Representation of the midline trunk, bilateral arms, and shoulders in the monkey postcentral somatosensory cortex. Exp Brain Res 123:315-322.

Taoka M, Toda T, Iriki A, Tanaka M, Iwamura Y (2000) Bilateral receptive field neurons in the hindlimb region of the postcentral somatosensory cortex in awake macaque monkeys. Exp Brain Res 134:139-146.

Wang X, Merzenich MM, Sameshima K, Jenkins WM (1995) Remodelling of hand representation in adult cortex determined by timing of tactile stimulation. Nature 378:71-75.

Welles JF (1976) A comparative study of manual prehension in anthropoids. Saugetierkundliche Mittielungen 24:26-38.

Wong-Riley M (1979) Changes in the visual system of monocularly sutured or enucleated cats demonstrable with cytochrome oxidase histochemistry. Brain Res 171:11-28.

Xerri C, Merzenich MM, Peterson BE, Jenkins W (1998) Plasticity of primary somatosensory cortex paralleling sensorimotor skill recovery from stroke in adult monkeys. J Neurophysiol 79:2119-2148.

Xerri C, Merzenich MM, Jenkins W, Santucci S (1999) Representational plasticity in cortical area $3 \mathrm{~b}$ paralleling tactual-motor skill acquisition in adult monkeys. Cereb Cortex 9:264-276. 